\title{
Learning about Quantum Gravity with a Couple of Nodes ${ }^{\star}$
}

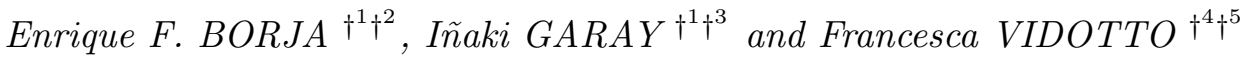 \\ $\dagger^{1}$ Institute for Theoretical Physics III, University of Erlangen-Nürnberg, \\ Staudtstraße 7, 91058 Erlangen, Germany \\ E-mail: efborja@theorie3.physik.uni-erlangen.de, igael@theorie3.physik.uni-erlangen.de \\ $\dagger^{2}$ Departamento de Física Teórica and IFIC, Centro Mixto Universidad de Valencia-CSIC, \\ Facultad de Física, Universidad de Valencia, Burjassot-46100, Valencia, Spain \\ $\dagger^{3}$ Departamento de Física Teórica, Universidad del País Vasco, \\ Apartado 644, 48080 Bilbao, Spain \\ $\dagger^{4}$ Laboratoire de Physique Subatomique et de Cosmologie, \\ 53 rue des Martyrs, 38026 Grenoble, France \\ $t^{5}$ Centre de Physique Théorique de Luminy, Case 907, 13288 Marseille, France \\ E-mail:vidotto@cpt.univ-mrs.fr
}

Received October 08, 2011, in final form March 12, 2012; Published online March 25, 2012

http://dx.doi.org/10.3842/SIGMA.2012.015

\begin{abstract}
Loop Quantum Gravity provides a natural truncation of the infinite degrees of freedom of gravity, obtained by studying the theory on a given finite graph. We review this procedure and we present the construction of the canonical theory on a simple graph, formed by only two nodes. We review the $\mathrm{U}(N)$ framework, which provides a powerful tool for the canonical study of this model, and a formulation of the system based on spinors. We consider also the covariant theory, which permits to derive the model from a more complex formulation, paying special attention to the cosmological interpretation of the theory.
\end{abstract}

Key words: discrete gravity; canonical quantization; spinors; spinfoam; quantum cosmology

2010 Mathematics Subject Classification: 83C27; 83C45; 83C60; 83F05

\section{Introduction to few-nodes models}

It's a long way to quantum gravity, and the way is not unique. We do not yet have a complete quantization of the gravitational field. Different proposals have been explored, and we are assisting to the convergence of some of them into a unique coherent picture, that takes the name of Loop Quantum Gravity (LQG) [85, 89, 102].

The state space of LQG, $H_{\mathrm{LQG}}$, admits subspaces that are determined by graphs $\Gamma$, whose physical meaning we discuss below. In [96] the idea was put forward to study the truncation of the full quantum theory on a very simple graph: a graph formed by only two nodes. This truncation, it was argued, can be sufficient to study cosmology. The idea has since been developed in various directions. First, the physical approximation involved in this truncation has become more clear. Second, the relation between the degrees of freedom captured by this "dipole" graph and the degree of freedom of Bianchi XI has been clarified.

More importantly, the "dipole" truncation has proven to be a natural context for developing the $\mathrm{U}(N)$ formalism, a powerful mathematical language for controlling the mathematical struc-

\footnotetext{
*This paper is a contribution to the Special Issue "Loop Quantum Gravity and Cosmology". The full collection is available at http://www.emis.de/journals/SIGMA/LQGC.html
} 
ture of the quantum states of geometry, especially in the homogeneous and isotropic context, and to suggest the form of the Hamiltonian.

Finally, the "dipole" graph has represented the starting point for deriving cosmological amplitudes from the covariant spinfoam theory, opening the way to the use of richer graphs.

In this article we review these different directions of research opened by the study of the "dipole" graph. We begin, below, by discussing the physical meaning of the graph. We discuss the original Hamiltonian quantization of dipole in the context of cosmology in Section 2, then the $\mathrm{U}(N)$ formalism in Section 3, and finally the spinfoam application in Section 4.

\subsection{Why graphs?}

Let us begin by discussing how a truncation can appear in quantum gravity, and how it is related to graphs.

Discrete gravity (1961). The essential idea behind the graph truncation can be traced to Regge calculus [79], which is based on the idea of approximating spacetime with a triangulation, where the metric is everywhere flat except on the triangles. On a fixed spacelike surface, Regge calculus induces a discrete 3 -geometry defined on a $3 \mathrm{~d}$ triangulation, where the metric is everywhere flat except on the bones. The two-skeleton of the dual of this $3 \mathrm{~d}$ cellular decomposition is a graph $\Gamma$, obtained by taking a point (a "node") inside each cell, and connecting it to the node in an adjacent cell by a link, puncturing the triangle shared by the two cells. These are the graph we are considering here. More precisely, we will consider some generalizations of this construction, where the cellular decomposition is not necessarily a triangulation and the geometry can be more discontinuous than a Regge geometry.

Spinnetworks (1971). In Loop Quantum Gravity, the spinnetwork basis $\left|\Gamma, j_{\ell}, \nu_{n}\right\rangle[13$, $14,92]$ is an orthonormal basis that diagonalizes the area and volume operators. The states in this basis are labelled by a graph $\Gamma$ and two quantum numbers coloring it: a spin $j$ at each link $\ell$ and a volume eigenvalue $\nu$ at each node $n$. The (diff-invariant) Hilbert space $H_{\Gamma}$ obtained by considering only the states on the (abstract) graph $\Gamma$ is precisely the Hilbert space of an SU(2) Yang-Mills theory on this lattice. Penrose's "spin-geometry" theorem connects this Hilbert space with the description of the geometry of the cellular decomposition mentioned above: states in this Hilbert space admit a geometrical interpretation [76] as a quantum version of the 3-geometry (see [89] and [26]). That is, a Regge 3-geometry defined on a triangulation with dual graph $\Gamma$ can be approximated by semiclassical state in $H_{\Gamma}{ }^{1}$

Holonomies (1986). In the canonical quantization of General Relativity (GR), in order to implement Dirac quantization, it's convenient to choose the densitized inverse triad $E^{i a}$ (Ashtekar's electric field) and the Ashtekar-Barbero connection $A_{a}^{i}$ as conjugate variables [3], and then use the flux of $E^{i a}$ and the the holonomy $h_{\gamma}=\mathcal{P} \exp \left[\int_{\gamma} A_{a}^{i}\right]$, namely the parallel transport operator for $A$ along a path $\gamma$, as fundamental variables for the quantization. In the quantum theory, all relevant physical objects (partial obsevables [84]), for instance the operators for area and volume, have support only on these paths and their intersections. Considering a truncation of the theory amounts to restricting the choice of the observables to a finite subset. In particular, the holonomies can be taken along the links of the graph, and the densitized inverse triad can be smeared over the faces of the triangulation. This connects the holonomytriad variables to the discrete geometry picture.

The common point of these different derivations is $3 \mathrm{~d}$ coordinate gauge invariance, that has important consequences ${ }^{2}$. This invariance is the reason for the use of abstract graphs: it removes

\footnotetext{
${ }^{1}$ Generically, the geometry defined by the semiclassical states in $H_{\Gamma}$ can be more general than a Regge geometry $[55,94]$. Furthermore, the graphs can be dual to generic cellular decomposition which are not triangulations, or to a cellular decomposition subjected to some specific restrictions [60, 63, 65].

${ }^{2}$ The $3 \mathrm{~d}$ coordinate gauge invariance is the fundamental assumption of the LOST theorem [48, 67], that states the uniqueness of the representation in the LQG Hilbert space.
} 

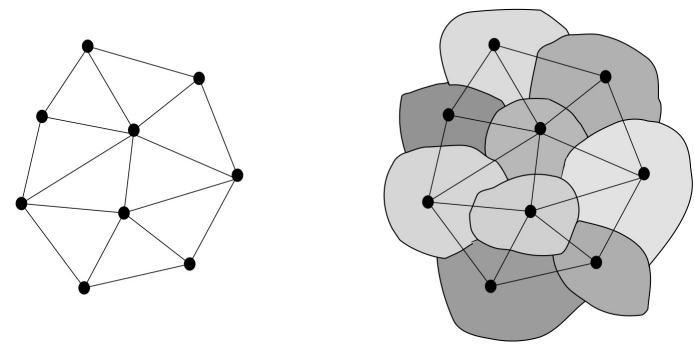

$\left|\Gamma, j_{l}, v_{n}\right\rangle$

Figure 1. To each node of a spinnetwork we can associate a "quantum of space".

the physical meaning of the location of the graph on the manifold. Therefore the graph we are considering is just a combinatorial object, that codes the adjacency of the nodes. Each node describe a quantum of space, and the graph describes the relations between different pieces of space. The Hilbert subspaces associated to distinct but topologically equivalent embedded graphs are identified $[90,91]$, and each graph space $h_{\Gamma}$ contains the Hilbert spaces of all the subgraphs.

\subsection{Doing physics with few nodes}

Full general relativity is recovered from Regge calculus only by moving to triangulations with an arbitrary large number of cells; the full LQG Hilbert space includes arbitrary large graphs; and the full set of observables cannot be restricted to holonomies and fluxes on a fixed graph. The restriction to a fixed triangulation or a fixed graph amounts only to a truncation of the theory, namely to disregarding an infinite number of degrees of freedom and cutting down the theory to an approximate theory with a finite number of degrees of freedom. But truncations are always needed in quantum field theory, in order to extract numbers from the theory. For instance, numerical lattice QCD calculations are performed on a finite lattice, and every given Feynman graph in QED involves only a finite number of particles, hence a truncation of the full theory ${ }^{3}$. A given truncation can provide a valuable approximation to the full theory only under certain conditions and in certain physical regimes. It is important to notice that in appropriate physical regimes even a low-order approximation can be effective. The Regge dynamics approximates the continuous GR dynamics when $R L^{2} \ll 1$ where $R$ is the curvature scale and $L$ the length of the Regge bones ${ }^{4}$. Therefore, as far as computing dynamics is concerned, a very rough triangulation can well approximate a near-flat spacetime. Very similarly, a first order Feynman diagram gives an excellent approximation to a scattering amplitude, even if the real spacetime trajectory of the particles is a smooth curved path, quite different from the piecewise straight path depicted in the Feynman diagram. Notice that since any given Feynman diagram involves a finite number $N$ of particle, the diagram is concretely defined on the subspace $H_{N}$ of Fock space spanned by the $n$-particle states with $n \leq N$.

In LQG, the analog of $H_{N}$ is the state space $H_{\Gamma}$ formed by the (diff-invariant) states on a given graph is a subspace of the full Hilbert space $H_{\mathrm{LQG}} \sim \lim _{\Gamma \rightarrow \infty} H_{\Gamma}[6,89]$. It has the structure $H_{\Gamma}=L^{2}\left[\mathrm{SU}(2)^{L} / \mathrm{SU}(2)^{n}\right]$, where $L$ the number of oriented links of $\Gamma, n$ is the number

\footnotetext{
${ }^{3}$ It is interesting to remark that these two very different truncation schemes (finite number of lattice sites in lattice QCD and finite number of particles in perturbative QED) end up merging in quantum gravity. This is because a lattice site is a region of space and in quantum gravity a region of space is quantum of the gravitational field, very much like a particle is a quantum of a quantum field. Therefore both truncations are reinterpreted in quantum gravity as expansions in the number of a quanta: a finite number of quanta of the gravitational field is described by a spin network, and is also a lattice in the sense of QCD, as shown by the structure of $H_{\Gamma}$ indicated below. This convergence of the different quantum field theory pictures of lattice QCD and perturbative QED is one of the most beautiful aspects of LQG.

${ }^{4}$ Namely when the Regge deficit angles, which code the curvature, are small.
} 
of nodes, and the group quotient is given by the gauge transformations at the nodes on the group elements on the links as in lattice gauge theory. It is therefore interesting to explore if we can compute physical quantities approximately working in $H_{\Gamma}$ instead of the full $\mathcal{H}_{\mathrm{LQG}}$, in the same spirit of Quantum Field Theory when one considers the Fock space for $N$ particles, instead of infinite particles. This approach to LQG is called graph expansion, and relies on the invariance under diffeomorphism, that we have just discussed above.

Truncating the theory to a given fixed $\Gamma$ corresponds to disregarding the states that need a "larger" graph to be defined, while all states that have support on graphs "smaller" than $\Gamma$ are already contained in $h_{\Gamma}{ }^{5}$

As the connection with Regge calculus shows, choosing a graph corresponds then to choosing an approximation for the system that we want to describe. Discretizing a continuous geometry by a given graph is nothing but coarse graning the theory. It is important to stress that the discreteness introduced by this process is different from the fundamental quantum discreteness of the theory. The first is the discreteness of the abstract graphs; the later is the discreteness of the spectra of the area and volume operator on each given $H_{\Gamma}$. Mistaking these two sources of discreteness has been a source of confusion in the literature ${ }^{6}$.

Another source of confusion in the literature is the confusion between two different expansion: the graph expansion and the semiclassical expansion (see Fig. 2). The first is obtained by a refinement of the graph, the second by a large-distance limit [89] on each graph. The first is an expansion valid in the regimes where a rough graph approximation is good (in particular at scales $L$ smaller that the curvature scale $R$ ); the second when we can disregard quantum effects, (in particular, at scales $L$ larger that the Planck scale $L_{P}$ ).

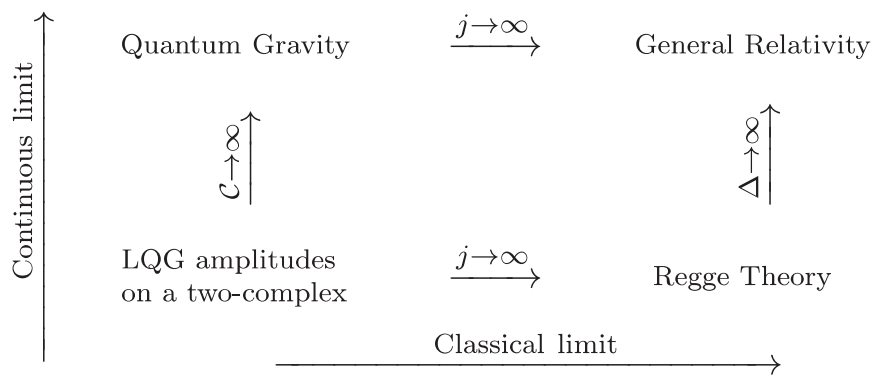

Figure 2. Continuous and classical limits in LQG.

This allows us to study two distinct limits in the theory:

Continuous limit. We are considering a discretized system with the further properties to be diff-invariant system. This lead to a peculiarity while taking the continuum limit, as discussed in [83]. In a theory like lattice QCD, this limit is achieved by sending the lattice spacing to zero and the coupling constant to its critical value. Here instead we just refine the graph, because the dynamics is not affected by the size of the discretization since coordinates are unphysical. This may have an important consequence: the discretization becomes nearly exact, the number of nodes behaves as an effective expansion parameter and the system may approach a regime where the theory is topological (Ditt-invariance regime [82]). This might happen in describing homogeneous and isotropic geometries [16, 17, 18, 19].

Classical limit. The fundamental discreteness of the theory is washed away when the largedistance limit of the theory is taken. This corresponds to considering large spins, namely $j \rightarrow \infty$. The classical theory is therefore recovered and the quantum parameters $\hbar$ and $\gamma$ (the Barbero-

\footnotetext{
${ }^{5}$ States with support on graph smaller than $\Gamma$ (subgraphs of $\Gamma$ ) are already included in $H_{\Gamma}$.

${ }^{6}$ This is analog to the case of an electromagnetic field in a box: the modes of the field are discrete and allow a truncation of the theory, but quantum discreteness is something else: it is given by the quantized energy of each mode.
} 

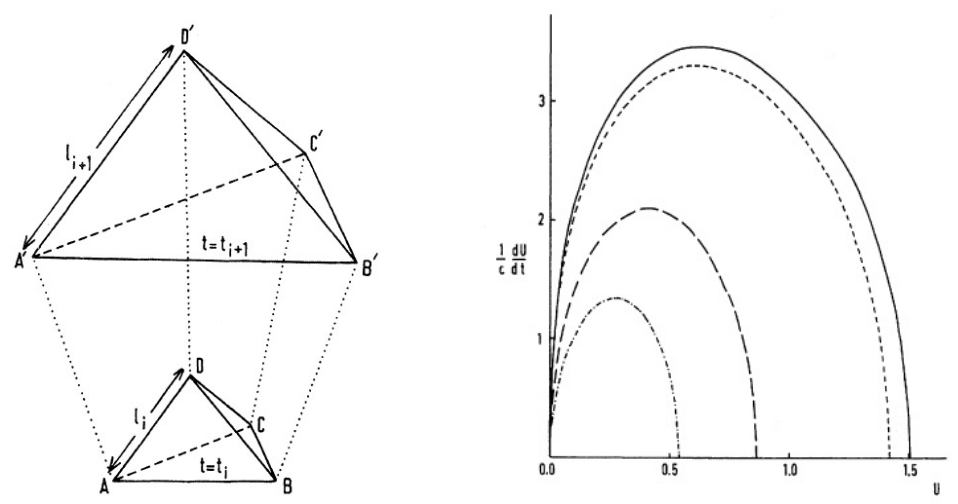

Figure 3. On the left, a $4 \mathrm{~d}$ building block of spacetime and, on the right, the evolution of 5, 16 and 500 of these building block (dashed lines), modeling a closed universe, compared whit the continuous analytic solution (solid line) [35]. The qualitative behavior of these universes, coded in the rate of change of the volume, is the same.

Immirzi parameter) disappear. Different ways to take the semi-classical limit, such that the quantum corrections would be under better control, are under study [73].

The classical continuous theory of General Relativity is recovered once both limits are taken. In quantum theory, these expansions have provided interesting insights on the full theory. (This will be addressed further while addressing the covariant theory in Section 4.)

The point of view that we are presenting in this review is that we may not necessarily be obliged to deal with very complicated graph in quantum gravity. Interesting physics can arise even by considering a simple graph, with few nodes, and comparing our results with classical discrete gravity (Regge calculus).

Notice that, in Regge calculus, few nodes are already enough to capture the qualitative behavior of the model. This is true for FLRW cosmologies. It has in fact been proven numerically [35] that the dynamics of a closed universe, with homogeneous and isotropic geometry, can be capture by 5, 16 and 600 nodes (these are the regular triangulations of a 3 -sphere) adapting the dynamics to these triangulations, and the only resulting difference is given by the scaling: having more tetrahedra, the growth is faster.

This review focuses on the construction of the theory when the graph is particularly small. In fact, we use here a minimal graph, given just by 2 nodes.

The main problem that we do not address in this review is how radiative corrections can affect the viability of the graph expansion. In a renormalizable quantum field theory we know that radiative corrections can be split into a finite part which is small, and therefore does not spoil the viability of the expansion, and a divergent part, which can be absorbed into a redefinition of fields and coupling constants. The same question arises in the present context: when refining the disctretization, do we generate corrections that are large and cannot be reabsorbed? This question, upon which relies the viability of the entire philosophy of the graph expansion has not been sufficiently studied yet $[66,78]$.

\subsection{The cosmological interpretation}

The graph expansion can be put in correspondence with a mode expansion of the gravitational field on a compact space [96]. (In the case of the dipole graph - see below - this correspondence has been worked out explicitly in [24].) The truncation of the theory on a graph provides a natural cut off of the infinite degrees of freedom of general relativity down to a finite number. Choosing a graph, we disregard the higher modes of this expansion. Therefore the truncation 
defines an approximation viable for gravitational phenomena where the ratio between the largest and the smallest relevant wavelengths in the boundary state is small.

Notice that this is neither an ultraviolet nor an infrared truncation, because the whole physical space can still be large or small. What is lost are not wavelengths shorter than a given length, but rather wavelengths $k$ times shorter than the full size of physical space, for some integer $k$. To understand the nature of this approximation, we can refer again to numerical lattice QCD. The number of lattice sites concretely needed for a numerical calculation is determined by the ratio between the smallest and largest wavelenghts involved in the phenomenon studied. For instance, for studying hadron masses [43], the relevant ratio is that determined by the hadron and quarks' sizes.

But the most striking example, where this kind of approximation applies, is given by cosmology itself. Modern cosmology is based on the cosmological principle, that says that the dynamics of a homogeneous and isotropic space approximates well our universe. The presence of inhomogeneities (which in the real universe are large and well beyond perturbation theory at small scales) can be disregarded at a first order approximation, where we consider the dynamics as described at the scale of the scale factor, namely the size of the universe. Thus our approximation depends on the scale factor, it is not just a large scale approximation: it depends on the ratio between the scale factor and the interaction that we want to consider. If we consider the dynamics of the whole universe, this ratio gives 1 , and an unique degree of freedom is concerned. We can then recover the full theory adding degrees of freedom one by one. We obtain an approximate dynamics of the universe, with a finite number of degrees of freedom. Postulating less symmetry, allows to add more degrees of freedom. So one can recover the full theory adding the degrees of freedom one by one. The specific choice of the truncation depends on the phenomena considered and the approximation desired.

In other words, working with a graph corresponds to choosing how many degrees of freedom we want to describe. A graph with a single degree of freedom is just one node: in a certain sense, this is the case of usual Loop Quantum Cosmology [12]. To add degrees of freedom, we add nodes and links with a coloring. These further degrees of freedom are a natural way to describe inhomogeneities and anisotropies [24, 96], present in our universe. When we ask the graph to give a regular cellular decomposition, node and links become indistinguishable, and we obtain back the unique FLRW degrees of freedom.
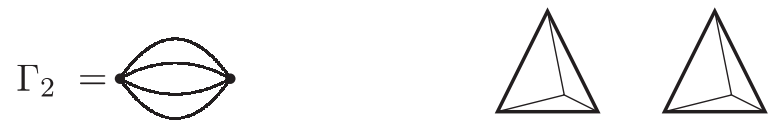

Figure 4. The "dipole" graph $\Gamma_{2}$ is given by 2 nodes and 4 links. It is dual to a triangulation of a 3 -sphere, where 2 tetrahedra are glued together by identifying their faces.

The easiest thing that can be done is to pass from $n=1$ to $n=2$ nodes. We choose to connect them by $L=4$ links, because in this way the dual graph will be two tetrahedra glued together, and this can be viewed as the triangulation of a 3 -sphere (Fig. 4). Note that we are not obliged to chose a graph corresponding to a triangulation, but this turn out to be very useful when we want to associate an intuitive interpretation to our model. In order to understand how this can be concretely use to do quantum gravity and quantum cosmology, we need to place on the graph the $\mathrm{SU}(2)$ variables.

\section{The Hamiltonian dipole}

In this section we review the original dipole construction, and the relation between its variables and those of the cosmological models. 


\subsection{LQG phase space}

Loop Quantum Gravity is a general covariant quantum field theory, where the $3 \mathrm{~d}$ coordinate gauge invariance is reflected in the choice of $\mathrm{SU}(2)$ as fundamental group. Let us start by associating the group element $h_{\ell} \in \mathrm{SU}(2)$ and a $\mathfrak{s} u(2)$ algebra element $E_{\ell}=E_{\ell}^{i} \tau_{i}$ where $\left\{\tau_{i} \mid i=\right.$ $1,2,3\}$ is a basis in $\mathfrak{s} u(2)$ to the links $\ell$ of a given graph $\Gamma$.

The cotangent boundle of $\mathrm{SU}(2)^{L}$ and its natural symplectic structure give the phase space of the theory: $h_{\ell}$ and $E_{\ell}$ are phase space variables with the conventional Poisson brackets structure of a canonical lattice SU(2) Yang-Mills theory, that is ${ }^{7}$

$$
\left\{h_{\ell}, h_{\ell^{\prime}}\right\}=0, \quad\left\{E_{\ell}^{i}, h_{\ell^{\prime}}\right\}=\delta_{\ell \ell^{\prime}} \tau^{i} h_{\ell}, \quad\left\{E_{\ell}^{i}, E_{\ell^{\prime}}^{j}\right\}=-\delta_{\ell \ell^{\prime}} \epsilon^{i j k} E_{\ell}^{k} .
$$

As in lattice QCD, a quantum representation of the observable algebra (2.1) is provided by the Hilbert space $\mathcal{H}_{\text {aux }}=L^{2}\left[\mathrm{SU}(2)^{L}, \mathrm{~d} h_{\ell}\right]$ where $\mathrm{d} h_{\ell}$ is the Haar measure. The operators $h_{\ell}$ are diagonal and the operators $E_{\ell}$ are the left invariant vector fields on each $\mathrm{SU}(2)$. The operators $E_{\ell^{-1}}$ turn then out to be the right invariant vector fields. These operators satisfy ${ }^{8}$ the

$$
\text { gauge constraint } \quad G_{n} \equiv \sum_{\ell} E_{\ell} \sim 0 \quad \forall \ell \in n,
$$

that can be seen as a closure condition on the cell dual to the node $n$.

The states that solve the gauge constraint (2.2) are labeled by $\mathrm{SU}(2)$ spinnetworks on the graph $\Gamma$. A basis of these is given by states $\left|j_{\ell}, \iota_{n}\right\rangle$, where $\ell=1, \ldots, L$ and $n=1, \ldots, N$ range over the links and the nodes of the graph. These are defined by

$$
\text { spinnetwork states } \quad \psi_{j_{\ell} \iota_{n}}\left(h_{\ell}\right) \equiv\left\langle h_{\ell} \mid j_{\ell}, \iota_{n}\right\rangle \equiv \otimes_{\ell} \Pi^{\left(j_{\ell}\right)}\left(h_{\ell}\right) \cdot \otimes_{n} \iota_{n},
$$

where $\Pi^{(j)}(U)$ are the matrix elements of the spin- $j$ representation of $\mathrm{SU}(2)$ and "." indicates the contraction of the indices of these matrices with the indices of the intertwiners $\iota_{n}$ dictated by the graph $\Gamma$. For details, see $[2,85,102]$.

Operators for area and volume can be constructed in terms of $E_{\ell}$. For each link $\ell$ we can associate the area of the face punctured by the link in the dual cellular decompositon

$$
\text { area } \quad A_{\ell}=\sqrt{E_{\ell} E_{\ell}}=8 \pi \gamma \ell_{\mathrm{Pl}}^{2} \sqrt{j_{\ell}\left(j_{\ell}+1\right)},
$$

where $\gamma$ is the Barbero-Immirzi parameter, and for each node we can associate the volume of the cell on which the node $n$ is sitting. The expression for a generic $n$-valent node is available but complicate [26], while in the simplest 4-valent case the expression becomes just

$$
\text { volume }^{2} \quad V_{n}^{2}=\frac{1}{4} \sum_{\ell \ell^{\prime} \ell^{\prime \prime} \in n} \operatorname{Tr}\left[E_{\ell} E_{\ell^{\prime}} E_{\ell^{\prime \prime}}\right]=\operatorname{Tr}\left[E_{\ell} E_{\ell^{\prime}} E_{\ell^{\prime \prime}}\right]
$$

where we have chosen the links $\left\{\ell, \ell^{\prime}, \ell^{\prime \prime}\right\}$ to have positive orientation. Notice that the sum over the four unordered triplets of distinct links drops because of (2.2). The total volume will be just $V_{\Gamma}=\sum V_{n} \forall n \subset \Gamma$. The dynamics is governed by the Hamiltonian constraint. Recall that the Hamiltonian constraint for general relativity can be written as [102]:

$$
C_{\text {grav }}=\int_{S^{3}} \mathrm{~d}^{3} x \epsilon_{k}^{i j} E^{a i} E^{b j} F_{a b}^{k}-2\left(1+\gamma^{2}\right) \int_{S^{3}} \mathrm{~d}^{3} x E^{a i} E^{b j} K_{[a}^{i} K_{b]}^{j}:=C+C_{\gamma},
$$

\footnotetext{
${ }^{7}$ Here we have assumed $\ell^{\prime} \neq \ell^{-1}$. If $\ell^{\prime}=\ell^{-1}$, the Poisson brackets are obtained using the equations $h_{\ell^{-1}}=U_{\ell}^{-1}$ and the algebra element $E_{\ell^{-1}}=-h_{\ell}^{-1} E_{\ell} h_{\ell}$.

${ }^{8}$ Notice that these variables transform properly under internal gauge transformations. In fact we can write $G[\lambda]:=2 \sum_{n} \operatorname{Tr}\left[\lambda_{n} G_{n}\right]$ where $\lambda_{n} \in s u(2)$, the infinitesimal gauge transformation of $h_{\ell}$ is $\delta h_{\ell}=\left\{h_{\ell}, G[\lambda]\right\}=$ $\lambda_{n_{1}} h_{\ell}-h_{\ell} \lambda_{n_{2}}$ where $\left(n_{1}, n_{2}\right)$ are respectively the source and the target of of the link $\ell$.
} 
where $K_{a}^{i}$ is the extrinsic curvature, and where we have set the lapse equal to the total 3-volume. Let us concentrate for the moment on the first term. If the discretization is appropriately chose before the quantization, we can write the first term of the Hamiltonian constraint by approxmating the field-strength tensor $F_{a b}^{k}$ with the holomy $h_{\ell \ell^{\prime}}$ such that

$$
C=\sum_{n} C_{n} \quad \text { with } \quad C_{n}=\sum_{\ell \ell^{\prime} \in n} \operatorname{Tr}\left[h_{\ell \ell^{\prime}} E_{\ell^{\prime}} E_{\ell}\right] \sim 0 .
$$

This form was suggested in the early day of LQG [93] and is nowadays exploited in loop cosmology $[5,12]$.

To complete our construction, at each node we couple a scalar field $\phi_{n}$, with conjugate momentum $p_{\phi_{n}}$, that work as a family of multifingered "clock" variables $[1,4,5,8]$. We need to introduce this physical clock because otherwise we would not be able to keep track of evolution in a background-independent manner [38, 39, 40, 84, 86, 88]. The introduction of a scalar field provides also a simplified manner to model the matter content of the universe. The generalization to a more realistic description is straightforward: here we will choose an ultralocal scalar field, but one can add the spacial derivative terms in the Hamiltonian of the matter field, and the description of Yang-Mills and fermion fields is particularly well-adapted to this formalism $[2,85,102]$.

Therefore the total Hamiltonian constraint is

$$
C_{\text {tot }}=\sum_{n} \sum_{\ell \ell^{\prime} \in n}\left(\operatorname{Tr}\left[h_{\ell \ell^{\prime}} E_{\ell^{\prime}} E_{\ell}\right]+C_{\gamma}+4 \pi G p_{\phi_{n}}^{2}\right) \sim 0
$$

where $G$ is the Newton constant, determining the matter-gravity coupling. With a scalar field, the Hilbert space becomes $\mathcal{H}_{\text {aux }}=L_{2}\left[\mathrm{SU}(2)^{L}, d h_{\ell}\right] \otimes L_{2}\left[R^{n}\right]$, with a (generalized) basis $\left|j_{\ell}, \nu_{n}, \phi_{n}\right\rangle$ and the states can be written in the form

$$
\psi\left(j_{\ell}, \nu_{n}, \phi_{n}\right) \equiv\left\langle j_{\ell}, \nu_{n}, \phi_{n} \mid \psi\right\rangle
$$

In this basis the operator $\phi_{n}$ is diagonal while $p_{\phi_{n}}=-i \frac{\partial}{\partial \phi_{n}}$.

\subsection{Dipole cosmology}

Consider the simple case obtained by taking $n=2$ and the natural triangulation of the a threesphere $S^{3}$ obtained by gluing two tetrahedra by all their faces, as in Fig. 4. This represents a finite dimensional truncation of LQG and describes the Bianchi IX Universe plus six inhomogeneous degrees of freedom [24]. The gravitational variables are $\left(h_{\ell}, E_{\ell}\right), \ell=1,2,3,4$. We have two Hamiltonian constraints, whose algebra is naturally closed given the simplicity of the system considered. The $\mathrm{SU}(2)$ symmetry structure enters twice in our description: not only in the discretization of the Ashtekar-Barbero variables, but also in order to add the inhomogeneities.

The gravitational Hilbert space is $L^{2}\left[\mathrm{SU}(2)^{4} / \mathrm{SU}(2)^{2}\right]$ and a basis of spinnetwork states that solve the gauge constraint is given by the states $\left|j_{\ell}, \iota_{n}\right\rangle=\left|j_{1}, j_{2}, j_{3}, j_{4}, \iota_{1}, \iota_{2}\right\rangle$. The action of one gravitational Hamiltonian constraint on a state gives

$$
\tilde{C}\left|j_{\ell}, \iota_{n}\right\rangle=\sum_{\ell \ell^{\prime}} C_{\ell \ell^{\prime}}\left|j_{\ell}, \iota_{n}\right\rangle
$$

where each term of the sum comes from one of the terms in the sum in $\ell$ and $\ell^{\prime}$ in (2.4). More explicitly, we have

$$
C_{12}\left|j_{1}, j_{2}, j_{3}, j_{4}, \iota_{1}, \iota_{2}\right\rangle=\sum_{\epsilon, \delta= \pm 1} C_{j_{f} \iota_{1} \iota_{2}}^{\epsilon \delta \iota_{1}^{\prime} \iota_{2}^{\prime}}\left|j_{1}+\frac{\epsilon}{2}, j_{2}+\frac{\delta}{2}, j_{3}, j_{4}, \iota_{1}^{\prime}, \iota_{2}^{\prime}\right\rangle
$$


because the operator $U_{12}=U_{1} U_{2}^{-1}$ in (2.4) multiplies the terms $\Pi^{j_{1}}\left(U_{1}\right)$ and $\Pi^{j_{2}}\left(U_{2}\right)$ and

$$
U \Pi^{j}(U)=\Pi^{1 / 2}(U) \Pi^{j}(U)=c_{+} \Pi^{j+1 / 2}(U)+c_{-} \Pi^{j-1 / 2}(U) .
$$

The matrix elements $C_{j \ell_{1} \iota_{2}}^{\epsilon \delta \iota_{1}^{\prime} \iota_{2}^{\prime}}$ can be computed with a straightforward exercise in recoupling theory from (2.4) (and from the Hamiltonian that makes use of the "Thiemann's trick" with some more algebra). In a different notation, in terms of the wave function components, we can write

$$
\tilde{C} \psi\left(j_{\ell}, \iota_{n}\right)=\sum_{\epsilon_{j}=0, \pm 1} C_{j_{\ell} \iota_{n}}^{\epsilon_{\ell} \iota_{n}^{\prime}} \psi\left(j_{\ell}+\frac{\epsilon_{\ell}}{2}, \iota_{n}^{\prime}\right),
$$

where $C_{j_{\ell} \iota_{n}}^{\epsilon_{j} \iota_{n}^{\prime}}$ vanishes unless $\epsilon_{\ell}=0$ for two and only two of the four $j$ 's. The scalar field variables are $\phi_{1}, \phi_{2}$. Taking these into account leads to the wave functions $\psi\left(j_{\ell}, \iota_{n}, \phi_{n}\right)$, and (2.5) gives the dynamical equations

$$
\begin{aligned}
& \left(\frac{\partial^{2}}{\partial \phi_{1}^{2}}+\frac{\partial^{2}}{\partial \phi_{2}^{2}}\right) \psi\left(j_{\ell}, \iota_{n}, \phi_{n}\right)=\frac{2}{\kappa} \sum_{\epsilon_{\ell}=0, \pm 1} C_{j_{\ell} \iota_{n}}^{\epsilon_{\ell} \iota_{n}^{\prime}} \psi\left(j_{\ell}+\frac{\epsilon_{j}}{2}, \iota_{n}^{\prime}, \phi_{n}\right), \\
& \frac{\partial^{2}}{\partial \phi_{1}^{2}} \psi\left(j_{\ell}, \iota_{n}, \phi_{n}\right)=\frac{\partial^{2}}{\partial \phi_{2}^{2}} \psi\left(j_{\ell}, \iota_{n}, \phi_{n}\right) .
\end{aligned}
$$

The coefficients $C$ can be computed explicitly from recoupling theory. They vanish unless two $\epsilon_{\ell}$ 's are zero. Equations (2.6), (2.7), defined on Hilbert space $\mathcal{H}_{2}=L_{2}\left[\mathrm{SU}(2)^{4} / \mathrm{SU}(2)^{2}\right] \otimes$ $L_{2}\left[R^{2}\right]$ define a quantum cosmological model which is just one step out of homogeneity.

\subsubsection{Born-Oppenheimer approximation and LQC}

We now ask if and how LQC is contained in the model defined above. The state space $H_{2}$ contains a subspace that could be identified as a homogeneous universe. This is the subspace $H^{\text {hom }} \subset H_{2}$ spanned by the states $\left|j, j, j, j, \iota_{j}, \iota_{j}, \phi, \phi\right\rangle$ where $\iota_{j}$ is the eigenstate of the volume that better approximates the volume of a classical tetrahedron whose triangles have area $j$. However, the dynamical equations (2.6), (2.7) do not preserve this subspace. This is physically correct, because the inhomogeneous degrees of freedom cannot remain sharply vanishing in quantum mechanics, due to Heisenberg uncertainty. Therefore it would be wrong to search for states that reproduce LQG exactly, within this model. In which sense then can a quantum homogeneous cosmology make sense?

The answer should be clear thinking to the meaning of the cosmological principle, that is at the base of every cosmological model. The cosmological principle is the hypothesis that in the theory there is a regime where the inhomogeneous degrees of freedom do not affect too much the dynamics of the homogeneous degrees of freedom, and that the state of the universe happens to be within such a regime. In other words, the homogeneous degrees of freedom can be treated as "heavy" degrees of freedom, in the sense of the Born-Oppenheimer approximation, and the inhomogeneous one can be treated as "light" ones. Let us therefore separate explicitly the two sets of degrees of freedom. This can be done as follows.

First, change variables from the group variables $h_{\ell} \in \mathrm{SU}(2)$ to algebra variables $A_{\ell} \in \mathfrak{s} u(2)$, defined by $\exp A_{\ell}=h_{\ell} \cdot{ }^{9}$ Following what is done in Loop Quantum Cosmology [4], let us fix a fiducial $\mathfrak{s} u(2)$ element $\omega_{\ell} \in \mathfrak{s} u(2)$ for each link $\ell$. We choose for simplicity a fiducial connection normalized as $\left|\omega_{\ell}\right|=1$, and such that the four vectors $\omega_{\ell}$ are normal to the faces of a regular tetrahedron centered at the origin of $\mathfrak{s} u(2) \sim R^{3}$. Our variables can be decomposed into

$$
A_{\ell}=c \omega_{\ell}+a_{\ell}, \quad E_{\ell}=p \omega_{\ell}+e_{\ell} .
$$

\footnotetext{
${ }^{9}$ This is only a convenient rewriting of the holonomies, not really a return of the connection as main variable.
} 
In order to fix this decomposition uniquely, we impose the following conditions: $p$ has to be determined by the total volume $V=p^{3 / 2}$, and $c$ should be its conjugate variable so that

$$
\{c, p\}=\frac{8}{3} \pi \gamma G \text {. }
$$

The variable $c$ can then be identified at the classical level with the scalar coefficients multiplying respectively the extrinsic and intrinsic curvature, namely we have $c \sim \gamma \dot{a}+1$ as in LGC, where $\dot{a}$ is the time derivative of the scale factor. We also define $\Delta V=V_{2}-V_{1}$, so that $V_{1,2}=\frac{1}{2}(V \pm \Delta V)$. In the quantum theory, $E_{\ell}$ turns out to be a left invariant vector field, call it $L_{\ell}$, so that (2.8) yields the decomposition

$$
L_{\ell}=\omega_{\ell} \frac{\partial}{\partial c}+\tilde{L}_{\ell}
$$

where $\tilde{L}_{\ell} c=0$. Inserting this decomposition into the Hamiltonian constraint (2.4) gives

$$
\tilde{C}_{n}=\sum_{\ell \ell^{\prime} \in t} \operatorname{Tr}\left[e^{c \omega_{\ell}-a_{\ell}} e^{-c \omega_{\ell^{\prime}}-a_{\ell^{\prime}}}\left(\omega_{\ell^{\prime}} \frac{\partial}{\partial c}+\tilde{L}_{\ell}\right)\left(\omega_{\ell} \frac{\partial}{\partial c}+\tilde{L}_{\ell^{\prime}}\right)\right] .
$$

Let us now decompose this constraint into two parts, the first of which depends only on the homogeneous variable $c$. This can be done keeping only the first term of the expansion of the exponentials in $a_{\ell}$ and $a_{\ell^{\prime}}$, and only the $V$ term in the volume term. That is, we write

$$
C_{n}=\frac{1}{2} C^{\mathrm{hom}}+C_{n}^{\mathrm{inh}},
$$

where

$$
\tilde{C_{n}}=\sum_{\ell \ell^{\prime} \in t} \operatorname{Tr}\left[e^{c \omega_{\ell}} e^{-c \omega_{\ell^{\prime}}}\left(\omega_{\ell^{\prime}} \frac{\partial}{\partial c}\right)\left(\omega_{\ell} \frac{\partial}{\partial c}\right)\right] .
$$

The interpretation of this spilt is transparent: $C^{\text {hom }}$ gives the gravitational energy in the homogeneous degree of freedom, while $C_{n}^{\text {inh }}$ gives the sum of the energy in the inhomogeneous degrees of freedom and the interaction energy between the two sets of degrees of freedom. Finally, we write the homogeneous variable $\phi=\phi_{1}+\phi_{2}$ and $\phi_{-}=\phi_{1}-\phi_{2}$.

Following Born and Oppenheimer, let us now make the hypothesis that the state can be rewritten in the form

$$
\psi\left(U_{\ell}, \phi_{n}\right)=\psi_{\text {hom }}(c, \phi) \psi_{\operatorname{inh}}\left(c, \phi ; a_{\ell}, \phi_{-}\right),
$$

where the variation of $\psi_{\text {inh }}$ with respect to $c$ and $\phi$ can be neglected at first order. Here $\psi_{\text {hom }}$ represents the quantum state of the homogeneous cosmological variables, while $\psi_{\text {inh }}$ represents the quantum state of the inhomogeneous fluctuations over the homogeneous background $(c, \phi)$. Inserting the Born-Oppenheimer ansatz (2.10) into the Hamiltonian constraint equation, and taking $N_{1}=N_{2}$, we have the equation

$$
\frac{\kappa}{2} \psi_{\mathrm{inh}} \frac{\partial^{2}}{\partial \phi^{2}} \psi_{\mathrm{hom}}+\frac{\kappa}{2} \psi_{\mathrm{hom}} \frac{\partial^{2}}{\partial \phi_{-}^{2}} \psi_{\mathrm{inh}}-\psi_{\mathrm{inh}} \tilde{C}^{\mathrm{hom}} \psi_{\mathrm{hom}}-\tilde{C}^{\mathrm{inh}} \psi_{\mathrm{hom}} \psi_{\mathrm{inh}}=0 .
$$

Dividing by $\psi_{\text {hom }} \psi_{\text {inh }}$ this gives

$$
\frac{\frac{\kappa}{2} \frac{\partial^{2}}{\partial \phi^{2}} \psi_{\mathrm{hom}}}{\psi_{\mathrm{hom}}}-\frac{\tilde{C}^{\mathrm{hom}} \psi_{\mathrm{hom}}}{\psi_{\mathrm{hom}}}=-\frac{\frac{\kappa}{2} \frac{\partial^{2}}{\partial \phi_{-}^{2}} \psi_{\mathrm{inh}}}{\psi_{\mathrm{inh}}}+\frac{\tilde{C}^{\mathrm{inh}} \psi_{\mathrm{hom}} \psi_{\mathrm{inh}}}{\psi_{\mathrm{hom}} \psi_{\mathrm{inh}}} .
$$


Since the left hand side of this equation does not depend on the inhomogeneous variables, there must be a function $\rho(c, \phi)$ such that

$$
\begin{aligned}
& \frac{\kappa}{2} \frac{\partial^{2}}{\partial \phi^{2}} \psi_{\text {hom }}-\tilde{C}^{\text {hom }} \psi_{\text {hom }}-\rho \psi_{\text {hom }}=0, \\
& \frac{\kappa}{2} \frac{\partial^{2}}{\partial \phi_{-}^{2}} \psi_{\text {inh }}+\frac{\tilde{C}^{\text {inh }} \psi_{\text {hom }} \psi_{\text {inh }}}{\psi_{\text {hom }}}=\rho \psi_{\text {inh }} .
\end{aligned}
$$

The second equation is the Schrödinger equation for the inhomogeneous modes in the background homogeneous cosmology $(c, \phi)$, where $\rho(c, \phi)$ plays the role of energy eigenvalue. The first equation is the quantum Friedmann equation for the homogeneous degrees of freedom $(c, \phi)$, corrected by the energy density $\rho(c, \phi)$ of the inhomogeneous modes. At the order zero of the approximation, where we disregard entirely the effect of the inhomogeneous modes on the homogeneous modes, we obtain

$$
\frac{\kappa}{2} \frac{\partial^{2}}{\partial \phi^{2}} \psi_{\text {hom }}=\tilde{C}^{\text {hom }} \psi_{\text {hom }}
$$

Let us now analyze the action of the operator $C^{\text {hom }}$, defined in (2.9). Notice that $c$ multiplies the generator of a $\mathrm{U}(1)$ subgroup of $\mathrm{SU}(2)^{4}$. Therefore it is a periodic variable $c \in[0,4 \pi]$. We can therefore expand the states $\psi_{\text {hom }}(c, \phi)$ in Fourier sum

$$
\psi_{\text {hom }}(c, \phi)=\sum_{\mu} \psi(\mu, \phi) e^{i \mu c / 2}
$$

where $\mu$ is an integer. The basis of states $\langle c \mid \mu\rangle=e^{i \mu c / 2}$ in the gravitational sector of the $\psi_{\text {hom's }}$ state space satisfies

$$
p^{\frac{3}{2}}|\mu\rangle=k \mu^{\frac{3}{2}}|\mu\rangle, \quad-4 \sin ^{2}(c / 2)|\mu\rangle=|\mu+2\rangle-2|\mu\rangle+|\mu-2\rangle,
$$

which we shall use below ${ }^{10}$. Here $k=\left(\frac{8 \pi G \gamma}{6}\right)^{\frac{3}{2}}$.

The homogeneous Hamiltonian constraint (2.9) can be rewritten as

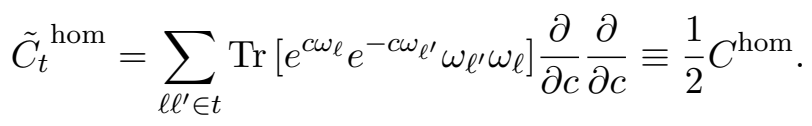

This can be rewritten as [24]

$$
\tilde{C}^{\text {hom }}=\sum_{\ell \ell^{\prime}} \operatorname{Tr}\left[\left(\cos \frac{c}{2} \mathbb{1}+2 \sin \frac{c}{2} \omega_{\ell}\right)\left(\cos \frac{c}{2} \mathbb{1}-2 \sin \frac{c}{2} \omega_{\ell^{\prime}}\right) \omega_{\ell^{\prime}} \omega_{\ell}\right] \frac{\partial^{2}}{\partial c^{2}}=\frac{17}{6}(\cos c-1) \frac{\partial^{2}}{\partial c^{2}} .
$$

The action of this operator on the states $\psi_{\text {hom }}(\mu, \phi)$ is therefore easily computed

$$
\tilde{C}^{\text {hom }} \psi_{\text {hom }}(v, \phi)=\frac{17}{6}\left[\mu^{2} \psi_{\text {hom }}(\mu+2, \phi)-\mu^{2} \psi_{\text {hom }}(\mu, \phi) \mu^{2} \psi_{\text {hom }}(\mu-2, \phi)\right] .
$$

Bringing everything together, the full equation (2.9) reads

$$
\begin{aligned}
C^{+}(\mu) \psi_{\mathrm{hom}}(\mu+2, \phi) & +C^{0}(\mu) \psi_{\mathrm{hom}}(\mu, \phi)+C^{-}(\mu) \psi_{\mathrm{hom}}(\mu-2, \phi) \\
+\frac{\partial^{2}}{\partial \phi^{2}} \psi_{\mathrm{hom}}(\mu, \phi) & =0,
\end{aligned}
$$

where the coefficient take the simple form $C^{ \pm}(\mu)=-\frac{1}{2} C^{0}(\mu)=\frac{\mu^{2}}{2 \kappa}$.

\footnotetext{
${ }^{10}$ The explicit relation between these states and the states in the inhomogeneous-model state-space is not straightforward, and will be investigated in detail elsewhere.
} 
Equation (2.11) has the structure of the LQC dynamical equation. Thus, a structure very similar to the one of LQC appears in the zero order Born-Oppenheimer approximation of a Loop Quantum Gravity quantization of a finite number of degrees of freedom of the gravitational field, truncated according to the approximation dictated by the cosmological principle.

We stress that we are not claiming here that the precise form of LQC is recovered. In particular, the two Hamiltonian operators might differ, because of possible different values of the coefficients. The exact relation between the two theories, and the possibility of adapting the quantization scheme to the precise form of LQC have not yet been investigated in detail.

Bounce. It is easy to see that, given the periodicity in $c$ in the gravitational part of the Hamiltonian constraint, the model we have presented leads to a bounce. A first study of this has been presented in [23]. In fact, the total Hamiltonian for the system reads

$$
\mathcal{H}=\mathcal{H}_{g}+\mathcal{H}_{m}=\frac{17}{6} p^{2}(\cos (c-\alpha)-1)+|p|^{3} \rho,
$$

where $\rho=\rho(t)$ is the matter energy density. Studying the equations of motion we obtain a modified Friedmann equation of the form

$$
\left(\frac{\dot{a}}{a}\right)^{2}=\left(\frac{\dot{p}}{2 p}\right)^{2} \propto \rho\left(1-\frac{\rho}{\rho_{c}}\right) .
$$

Here $\rho_{c}$, playing the role of the critical density at which the bounce occurs, is defined as $\rho_{c}=$ $17 /(3 p)[23]$. We notice that such a density depends on $p$, giving rise to incompatibility with a proper classical limit of the model [37]. This is a situation already found in the old models of LQC and we know it depends on a naive regularization of the Hamiltonian constraint. In LQC there is a preferred regularization, that corresponds with the so-called $\bar{\mu}$-scheme [9]: the critical density is a constant in this way, and its value depends on the minimal are gap provided by LQG, i.e. the minimal eigenvalue given by (2.3). At the moment we write, the implementation of this kind of regularization in the dipole model is under study. We refer the interested reader to [68], in particular for what concerns the implementation of the $\bar{\mu}$-scheme in the spinor framework, that will be introduced in Section 3 .

\subsubsection{Anisotropies and inhomogeneities}

In the previous treatment we have performed the Bohr-Oppenheimer approximation in such a way to extract the heavier degree of freedom, given by the homogeneous and isotropic one coded in the scale factor. On the other hand, we have already mentioned that, using the dipole graph as the base of our model, we are in a natural situation to accommodate more that a single degree of freedom. In fact, the Hilbert space of the dipole graph contains 6 degrees of freedom. These should code information about the presence of anisotropy and inhomogeneity, but haw can we relate them to a classical interpretation? In classical General Relativity, the maximal amount of anisotropy that a system with the topology of a 3-sphere can have, is described by the Bianchi IX model. This is usually charachterized by three scale factors $a_{I}=a_{I}(t)$, which identify three independent directions in the time evolution of the Cauchy surfaces. In the connection formalism, we have to consider three different connections $c_{I}=c_{I}(t)$ and momenta $p_{I}=p_{I}(t)$. Therefore the basic variables (2.8) in our model takes the form

$$
h_{\ell}=\exp \left(c^{I} \omega_{\ell}^{I} \tau_{I}\right), \quad E_{\ell}=p^{I} \omega_{\ell}^{I} \tau_{I} .
$$

Notice that here the holonomies (2.12) are simply group elements taken on the links of the dipole, without any specific orientation. The connection components are summed over and they are thus independent on the $I$-direction. This differs from the situation in the LQC Bianchi IX 
model [109], where the basic holonomies $h_{I}$ are computed along paths parallel to the three axis individuated by the anisotropies.

The anisotropies of the Bianchi IX model requires only three degrees of freedom. The remaining large-scale gravitational degrees of freedom captured by the dipole dynamics are necessarily inhomogeneous. Recall that in Section 1.3 we introduced the idea that these degrees of freedom can be added one by one as the terms of an expansion in modes. Therefore we consider here an expansion of the gravitational fields in tensor harmonics: the lowest modes should be the ones captured by the dipole dynamics. Luckily a similar mode expansion around Bianchi IX has been already studied by Regge and Hu using Wigner functions [61]. The Wigner functions $D_{\alpha^{\prime} \alpha}^{j}(g(x))$ determine a basis of functions of the symmetry-group of the model. Recall that we can use group elements $g(x)$ to coordinatize the physical space that has the $S^{3}$ topology.

Let us adapt this formalism to the first order variables we use. We start with the triads. We write a generic perturbed triad $E_{I}^{a}(x, t)$ as the sum of the background triad $e_{I}$ field and a perturbation

$$
E_{I}^{a}(x, t)=e_{I}^{a}(x)+\psi_{I J}(x, t) e_{J}^{a}(x) .
$$

We write this as a sum of components of definite $j$ and $\alpha$ quantum numbers

$$
\psi_{I J}(x, t)=\sum_{j \alpha} \psi_{I J}^{j \alpha}(x, t), \quad \text { where } \quad \psi_{I J}^{j \alpha}(x, t)=\sum_{\alpha^{\prime}=-j}^{j} \psi_{I J}^{j \alpha \alpha^{\prime}}(t) D_{\alpha^{\prime} \alpha}^{j}(g(x)) .
$$

The same can be done for the connection

$$
\omega_{a}^{I}(x) \rightarrow \tilde{\omega}_{a}^{I}(x, t)=\omega_{a}^{I}(x)+\varphi^{I J}(x, t) \omega_{a}^{J}(x) .
$$

Expanding this in components of definite $j$ and $\alpha$ quantum numbers gives

$$
\varphi^{I J}(x, t)=\sum_{j \alpha} \varphi_{j \alpha}^{I J}(x, t), \quad \text { where } \quad \varphi_{j \alpha}^{I J}(x, t)=\sum_{\alpha^{\prime}=-j}^{j} \varphi_{j \alpha \alpha^{\prime}}^{I J}(t) D_{\alpha^{\prime} \alpha}^{j}(g(x)) .
$$

The $\left(\varphi_{j \alpha \alpha^{\prime}}^{I J}(t), \psi_{j \alpha \alpha^{\prime}}^{I J}(t)\right)$ are the time-dependent expansion coefficients that capture the inhomogeneous degrees of freedom. They, are given by matrices in the internal indices $I, J$, labeled by the spin $j$ that runs from $j=1 / 2$ to all the semi-integers numbers, and the corresponding magnetic number $\alpha$.

We want to have the inhomogeneities determined by namely nine degrees of freedom coded in $\varphi_{\alpha}^{I}(t), \psi_{\alpha}^{I}(t)$. This is achieved by assuming that the matrices $\left(\varphi_{j \alpha \alpha^{\prime}}^{I J}(t), \psi_{j \alpha \alpha^{\prime}}^{I J}(t)\right)$ are diagonal in the internal indices $I, J$ and it is different from zero only for lowest nontrivial integer spin $j=1$ and for, say, $\alpha=0$. That is, we restrict to the components

$$
\varphi_{1,0, \alpha}^{I J}(t)=\delta^{I J} \varphi_{\alpha}^{I}(t), \quad \psi_{1,0, \alpha}^{I J}(t)=\delta^{I J} \psi_{\alpha}^{I}(t), \quad \alpha=-1,0,1 .
$$

The Gauss constraint reduces further the degrees of freedom to six, which is the number of degrees of freedom captured by the dipole variables. Therefore, we can interpret the six extra degrees of freedom of the dipole model (beyond anisotropies), as a description of the diagonal part of the lowest integer mode of the inhomogeneities. In this way the variables of the dipole model can be connected to the quantities $\left(\varphi_{\alpha}^{I}(t), \psi_{\alpha}^{I}(t)\right)$.

In order to complete the connection with the dipole variables, let us consider the fiducialalgebra elements $\omega_{f}^{I}$. these are perturbed as well, and as a consequence the 1-forms $\tilde{\omega}^{I}$ no longer satisfy the Maurer-Cartan structure equation $2 d \omega^{I}-\epsilon^{I}{ }_{J K} \omega^{J} \wedge \omega^{K}=0$. At first order, for a generic perturbation, let us define

$$
\tilde{\omega}_{f}^{I}=\frac{1}{2} \int_{f} \epsilon^{I}{ }_{J K} \tilde{\omega}^{J} \wedge \tilde{\omega}^{K}=\omega_{f}^{I}+\int_{f} \epsilon^{I} J K^{J} \omega^{J} \wedge \varphi^{K}=\omega_{f}^{I}+\sum_{j \alpha \alpha^{\prime}} \varphi_{j \alpha \alpha^{\prime}}^{K L}(t) \phi_{f K L}^{I j \alpha \alpha^{\prime}},
$$


where $\phi_{f K L}^{I j \alpha \alpha^{\prime}}=\int_{f} \epsilon^{I}{ }_{J K} D_{\alpha \alpha^{\prime}}^{j} \omega^{J} \wedge \omega^{L}$. In particular, if we restrict to the diagonal $j=1, \alpha=0$ case,

$$
\tilde{\omega}_{f}^{I}=\omega_{f}^{I}+\varphi_{\alpha}^{(I)}(t) \phi_{f, \alpha}^{(I)}, \quad \text { where } \quad \phi_{f, \alpha}^{I}=\int_{f} \epsilon_{J K}^{I} D_{0 \alpha}^{1} \omega^{J} \wedge \omega^{K}
$$

are fixed coefficients. Then the relation with the dipole variables can be written as

$$
h_{\ell}\left(c^{I}, \varphi_{\alpha}^{I}\right)=\exp \left(c^{I} \tilde{\omega}_{\ell}^{I} \tau_{I}\right) \exp \left(\alpha \omega_{\ell}^{I} \tau_{I}\right)
$$

which replaces (2.12). Similarly, we can write

$$
\tilde{E}_{\ell}^{I}=\int_{\ell}\left(e_{I}^{a}+\psi_{I}^{a}\right) \epsilon_{a b c} d x^{b} \wedge d x^{c}=E_{\ell}^{I}+2 \int_{\ell} \psi_{I J} \epsilon_{K L}^{J} \omega^{K} \wedge \psi^{L}=E_{\ell}^{I}+2 \sum_{j \alpha \alpha^{\prime}} \psi_{I J}^{j \alpha} \phi_{f, \alpha \alpha^{\prime}}^{J} .
$$

In particular, if we restrict to the diagonal $j=1, \alpha=0$ case,

$$
\tilde{E}_{\ell}^{I}=E_{\ell}^{I}+2 \psi_{I}^{\alpha} \phi_{f, \alpha}^{I} .
$$

Notice that now the Gauss constraint do not vanish identically. It can be split into two parts: the homogeneous and the inhomogeneous terms

$$
\mathcal{G}^{I}=\sum_{\ell} p^{(I)} \omega_{\ell}^{(I)}+2 \psi_{I}^{\alpha} \sum_{\ell} \phi_{f, \alpha}^{I} \approx 0
$$

The first part is the constraint which appears within the Bianchi IX framework and vanishes identically because of the Stokes theorem. The second gives three conditions on the inhomogeneous perturbations to the electric fields $\tilde{E}_{\ell}$.

\section{The $\mathrm{U}(N)$ framework}

A number of technical problems are still open in the canonical framework for LQG. Among these is to determine a form of the dynamics with a fully satisfactory semiclassical limit (but see the recent developments in [41]), and the difficulty of identifying a homogeneous sector yielding the Loop Quantum Cosmology (LQC) formalism. A novel point of view on these problems has been developed in a number of recent papers $[53,54,57]$. This is based on the identification of a $\mathrm{U}(N)$ symmetry in the Hilbert space of LQG intertwiners with $N$ legs and fixed total area. In this Section we illustrate the basis of this approach, called the $\mathrm{U}(N)$ framework, and we introduce the simplest nontrivial system where this approach prove useful. This system is again based on a 2-node graph as in the previous Section, but now with an arbitrary number $L$ of links. The relevant symmetry will be given by the group $\mathrm{U}(N)$, where $N=L[32,33,34]$. This framework allows us to introduce a specific dynamics for the 2-node graph and a convincing definition of homogeneity and isotropy. In this sense, the $\mathrm{U}(N)$ framework may prove a useful tool for addressing the problems mentioned above.

The $\mathrm{U}(N)$ framework has been developed in various directions. Recently, it was given a nice interpretation in terms of spinors, where intertwiner spaces can be reinterpreted as the product of the quantization of spinors model [70]. Other directions of research are the definition of coherent states [54], the reinterpretation of this framework in terms of holomorphic functions [34, 70], and the study of the simplicity constraints which appear in the spinfoam models [42]. In this review we focus on the basis of the $\mathrm{U}(N)$ framework, the complete treatment of the 2-node model and the possibility of understanding it as a classical system written in terms of spinors. 


\subsection{Introduction to the $\mathrm{U}(N)$ framework}

The $\mathrm{U}(N)$ symmetry defines a framework to investigate the structure of the Hilbert space of the intertwiners, which are the building blocks of spinnetwork states.

We consider first the space of $N$-valent intertwiners: the space of $\mathrm{SU}(2)$ invariant tensors of $N$ spins (SU(2) representations). Such intertwiners can be thought dually as a region of $3 \mathrm{~d}$ space with a (topologically) spherical boundary punctured by the $N$ legs of the intertwiners. The boundary surface is made of $N$ elementary patches, whose areas are determined by the spins carried by the intertwiner legs.

The Hilbert space of the intertwiner space for the group $\mathrm{SU}(2)$ is defined as

$$
\mathcal{H}_{j_{1}, \ldots, j_{N}} \equiv \operatorname{Inv}\left[V^{j_{1}} \otimes \cdots \otimes V^{j_{N}}\right]
$$

where $V^{j_{i}}$ are the irreducible representation spaces associated to the spin $j_{1}, \ldots, j_{N}$.

The key idea of the $\mathrm{U}(N)$ formalism is to consider the space formed by all the intertwiners with $N$ legs and fixed total sum of the spin numbers $J=\sum_{i} j_{i}$ (related with the total area of the boundary surface). That is

$$
\mathcal{H}_{N}^{(J)} \equiv \bigoplus_{\sum_{i} j_{i}=J} \mathcal{H}_{j_{1}, \ldots, j_{N}}
$$

It can be shown that the intertwiner space $\mathcal{H}_{N}^{(J)}$ carries an irreducible representation of $\mathrm{U}(N)$ [53]; and the full space $\mathcal{H}_{N} \equiv \bigoplus_{J} \mathcal{H}^{(J)}$ can be endowed with a Fock space structure with creation and annihilation operators compatible with the $\mathrm{U}(N)$ action [54]. In the following, we review the basics of this construction.

The first step to arrive to the $\mathrm{U}(N)$ framework is to make use of the well known Schwinger representation of the $\mathfrak{s} u(2)$ algebra. This representation consists on describing the generators of $\mathfrak{s} u(2)$ in terms of a pair of uncoupled harmonic oscillators. We introduce $2 N$ oscillators with operators $a_{i}, b_{i}$ with $i$ running from 1 to $N$ (a pair of uncoupled harmonic oscillators for each leg of the intertwiner), satisfying

$$
\left[a_{i}, a_{j}^{\dagger}\right]=\left[b_{i}, b_{j}^{\dagger}\right]=\delta_{i j}, \quad\left[a_{i}, b_{j}\right]=0
$$

The local $\mathfrak{s} u(2)$ generators acting on each leg $i$ are defined as quadratic operators

$$
J_{i}^{z}=\frac{1}{2}\left(a_{i}^{\dagger} a_{i}-b_{i}^{\dagger} b_{i}\right), \quad J_{i}^{+}=a_{i}^{\dagger} b_{i}, \quad J_{i}^{-}=a_{i} b_{i}^{\dagger}, \quad E_{i}=\left(a_{i}^{\dagger} a_{i}+b_{i}^{\dagger} b_{i}\right) .
$$

The $J_{i}$ 's constructed in this way satisfy the standard commutation relations of the $\mathfrak{s} u(2)$ algebra while the total energy $E_{i}$ is a Casimir operator

$$
\left[J_{i}^{z}, J_{i}^{ \pm}\right]= \pm J_{i}^{ \pm}, \quad\left[J_{i}^{+}, J_{i}^{-}\right]=2 J_{i}^{z}, \quad\left[E_{i}, \vec{J}_{i}\right]=0 .
$$

The operator $E_{i}$ is the total energy carried by the pair of oscillators $a_{i}, b_{i}$ and gives $2 j_{i}$, namely twice the spin, of the corresponding $\mathrm{SU}(2)$-representation. Indeed, we can easily express the standard $\mathrm{SU}(2)$ Casimir operator in terms of this energy

$$
\vec{J}_{i}^{2}=\frac{E_{i}}{2}\left(\frac{E_{i}}{2}+1\right)=\frac{E_{i}}{4}\left(E_{i}+2\right)
$$

In LQG the spin $j_{i}$ is related to the area associated to the leg $i$ of the intertwiner. Notice that in this context the most natural ordering of the area operator is the one given by the Casimir $E_{i} / 2$.

Our goal is to construct operators acting on the Hilbert space of intertwiners. In other words, we look for operators invariant under global SU(2) transformations generated by $\vec{J} \equiv \sum_{i} \vec{J}_{i}$. The 
key result, which is the starting point of the $\mathrm{U}(N)$ formalism, is that we can identify quadratic invariant operators acting on pairs of (possibly equal) legs $i, j[53,57]$

$$
E_{i j}=a_{i}^{\dagger} a_{j}+b_{i}^{\dagger} b_{j}, \quad E_{i j}^{\dagger}=E_{j i}, \quad F_{i j}=\left(a_{i} b_{j}-a_{j} b_{i}\right), \quad F_{j i}=-F_{i j} .
$$

These operators $E, F, F^{\dagger}$ form a closed algebra:

$$
\begin{aligned}
& {\left[E_{i j}, E_{k l}\right]=\delta_{j k} E_{i l}-\delta_{i l} E_{k j},} \\
& {\left[E_{i j}, F_{k l}\right]=\delta_{i l} F_{j k}-\delta_{i k} F_{j l}, \quad\left[E_{i j}, F_{k l}^{\dagger}\right]=\delta_{j k} F_{i l}^{\dagger}-\delta_{j l} F_{i k}^{\dagger},} \\
& {\left[F_{i j}, F_{k l}^{\dagger}\right]=\delta_{i k} E_{l j}-\delta_{i l} E_{k j}-\delta_{j k} E_{l i}+\delta_{j l} E_{k i}+2\left(\delta_{i k} \delta_{j l}-\delta_{i l} \delta_{j k}\right),} \\
& {\left[F_{i j}, F_{k l}\right]=0, \quad\left[F_{i j}^{\dagger}, F_{k l}^{\dagger}\right]=0 .}
\end{aligned}
$$

The commutators of the $E_{i j}$ operators form a $\mathfrak{u}(N)$-algebra (hence the name of the $\mathrm{U}(N)$ framework). The diagonal operators are equal to the energy on each leg, $E_{i i}=E_{i}$. The value of the total energy $E \equiv \sum_{i} E_{i}$ gives twice the sum of all spins $2 \times \sum_{i} j_{i}$, i.e. twice the total area.

The $E_{i j}$-operators change the energy/area carried by each leg, while still conserving the total energy, while the operators $F_{i j}$ (resp. $F_{i j}^{\dagger}$ ) decrease (resp. increase) the total area $E$ by 2

$$
\left[E, E_{i j}\right]=0, \quad\left[E, F_{i j}\right]=-2 F_{i j}, \quad\left[E, F_{i j}^{\dagger}\right]=+2 F_{i j}^{\dagger} .
$$

This suggests to decompose the Hilbert space of $N$-valent intertwiners into subspaces of fixed area

$$
\mathcal{H}_{N}=\bigoplus_{\left\{j_{i}\right\}} \operatorname{Inv}\left[\otimes_{i=1}^{N} V^{j_{i}}\right]=\bigoplus_{J \in \mathbb{N}} \bigoplus_{\sum_{i} j_{i}=J} \operatorname{Inv}\left[\otimes_{i=1}^{N} V^{j_{i}}\right]=\bigoplus_{J} \mathcal{H}_{N}^{(J)}
$$

where $V^{j_{i}}$ denote the Hilbert space of the irreducible $\mathrm{SU}(2)$-representation of spin $j_{i}$, spanned by the states of the oscillators $a_{i}, b_{i}$ with fixed total energy $E_{i}=2 j_{i}$.

It was proven in [53] that each subspace $\mathcal{H}_{N}^{(J)}$ of $N$-valent intertwiners with fixed total area $J$ carries an irreducible representation of $\mathrm{U}(N)$ generated by the $E_{i j}$ operators. The operators $E_{i j}$ allow to navigate from state to state within each subspace $\mathcal{H}_{N}^{(J)}$. On the other hand, the operators $F_{i j}, F_{i j}^{\dagger}$ allow to go from one subspace $\mathcal{H}_{N}^{(J)}$ to the next $\mathcal{H}_{N}^{(J \pm 1)}$, thus endowing the full space of $N$-valent intertwiners with a Fock space structure with creation operators $F_{i j}^{\dagger}$ and annihilation operators $F_{i j}$.

Finally, the whole set of operators $E_{i j}, F_{i j}, F_{i j}^{\dagger}$ satisfy quadratic constraints [32], $\forall i, j$ :

$$
\begin{aligned}
& \sum_{k} E_{i k} E_{k j}=E_{i j}\left(\frac{E}{2}+N-2\right), \\
& \sum_{k} F_{i k}^{\dagger} E_{j k}=F_{i j}^{\dagger} \frac{E}{2}, \quad \sum_{k} E_{j k} F_{i k}^{\dagger}=F_{i j}^{\dagger}\left(\frac{E}{2}+N-1\right), \\
& \sum_{k} E_{k j} F_{i k}=F_{i j}\left(\frac{E}{2}-1\right), \quad \sum_{k} F_{i k} E_{k j}=F_{i j}\left(\frac{E}{2}+N-2\right), \\
& \sum_{k} F_{i k}^{\dagger} F_{k j}=E_{i j}\left(\frac{E}{2}+1\right), \quad \sum_{k} F_{k j} F_{i k}^{\dagger}=\left(E_{i j}+2 \delta_{i j}\right)\left(\frac{E}{2}+N-1\right) .
\end{aligned}
$$

As already noticed in [32] and extended in [34], these relations look a lot like constraints on the multiplication of two matrices $E_{i j}$ and $F_{i j}$. We will explore this fact at the end of this section, but first we discuss the 2-node model from the $\mathrm{U}(N)$ perspective. 


\subsection{Hilbert space for the 2-node model}

We are going to establish the main steps to construct the Hilbert space of the 2-node model. In this case, we need to deal with two intertwiner spaces of $N$ legs each, as shown in Fig. 5 .

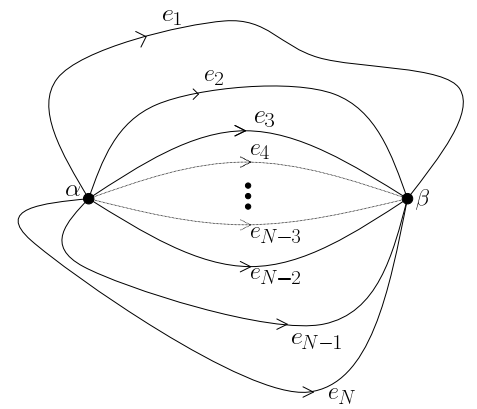

Figure 5. The 2-node graph: the two nodes $\alpha$ and $\beta$ are linked by $N=L$ links.

Naming the two nodes $\alpha$ and $\beta$, we have two intertwiner spaces attached respectively to $\alpha$ and $\beta$ with operators $E_{i j}^{(\alpha)}, F_{i j}^{(\alpha)}$ and $E_{i j}^{(\beta)}, F_{i j}^{(\beta)}$. The total Hilbert space of these uncoupled intertwiners is the tensor product of the two spaces of $N$-valent intertwiners

$$
\mathcal{H}_{\otimes 2}=\mathcal{H}_{N} \otimes \mathcal{H}_{N}=\bigoplus_{J_{\alpha}, J_{\beta}} \mathcal{H}_{N}^{\left(J_{\alpha}\right)} \otimes \mathcal{H}_{N}^{\left(J_{\beta}\right)}=\bigoplus_{\left\{j_{i}^{\alpha}, j_{i}^{\beta}\right\}} \mathcal{H}_{j_{1}^{\alpha}, \ldots, j_{N}^{\alpha}}^{\alpha} \otimes \mathcal{H}_{j_{1}^{\beta}, \ldots, j_{N}^{\beta}} .
$$

This space carries two decoupled $\mathrm{U}(N)$-actions, one acting on the intertwiner space associated to the node $\alpha$ and the other acting on $\beta$. However, the two intertwiner spaces are not independent. There are matching conditions unique $\mathrm{SU}(2)$ representation, thus the spin on that link must be the same seen from $\alpha$ or $\beta$, i.e. $j_{i}^{\alpha}=j_{i}^{\beta}$. This translates into the fact that the oscillator energy for $\alpha$ on the leg $i$ must be equal to the energy for $\beta$ on its $i$-th leg

$$
\mathcal{E}_{i} \equiv E_{i}^{(\alpha)}-E_{i}^{(\beta)}=0 .
$$

Obviously, this set of conditions is stronger than requiring that the total area of $\alpha$ is the same as $\beta$, even though this is a necessary condition. Then, the Hilbert space of spinnetwork states on the 2-node graph is much smaller than the decoupled Hilbert space $\mathcal{H}_{\otimes 2}$

$$
{ }^{2} \mathcal{H} \equiv \bigoplus_{\left\{j_{i}\right\}} \mathcal{H}_{j_{1}, \ldots, j_{N}}^{(\alpha)} \otimes \mathcal{H}_{j_{1}, \ldots, j_{N}}^{(\beta)} .
$$

In order to define consistent operators acting on ${ }^{2} \mathcal{H}$, we have to check that they commute (at least weakly) with the matching conditions $\mathcal{E}_{i}$, in addition to the condition that they need to be invariant under global $\mathrm{SU}(2)$ transformations. It is possible to construct such operators deforming consistently the boundary between $\alpha$ and $\beta$. We introduce the following operators, mixing actions on the two nodes as required

$$
e_{i j} \equiv E_{i j}^{(\alpha)} E_{i j}^{(\beta)}, \quad f_{i j} \equiv F_{i j}^{(\alpha)} F_{i j}^{(\beta)}, \quad f_{i j}^{\dagger} \equiv F_{i j}^{(\alpha) \dagger} F_{i j}^{(\beta) \dagger},
$$

and we check that they commute with the matching conditions

$$
\forall i, j, k, \quad\left[\mathcal{E}_{k}, e_{i j}\right]=\left[\mathcal{E}_{k}, f_{i j}\right]=0 .
$$

Calling $E=\sum_{i} E_{i}^{(\alpha)}=\sum_{i} E_{i}^{(\beta)}$ the operator giving (twice) the total boundary area on our Hilbert space ${ }^{2} \mathcal{H}$ satisfying the matching conditions, the operators $e_{i j}$ 's preserve the boundary area while the $f_{i j}$ 's will (as it is expected by construction) modify it

$$
\left[E, e_{i j}\right]=0, \quad\left[E, f_{i j}\right]=-2 f_{i j}, \quad\left[E, f_{i j}^{\dagger}\right]=+2 f_{i j}^{\dagger} .
$$


More precisely, the operator $e_{i j}$ increases the spin on the $i$-th link by $+\frac{1}{2}$ and decreases the spin of the $j$-th link. The operator $f_{i j}$ decreases both spins on the links $i$ and $j$, while its adjoint $f_{i j}^{\dagger}$ increases both spins by $\frac{1}{2}$. These operators generate the deformations of the boundary surface, consistently with both the $\mathrm{SU}(2)$ gauge invariance and the matching conditions imposed by the graph combinatorial structure. They are natural building blocks for the dynamics of spin network states on the 2-node graph.

Nevertheless, the operators $e_{i j}, f_{i j}$ and $f_{i j}^{\dagger}$ are not enough to generate the whole Hilbert space ${ }^{2} \mathcal{H}$ of spin network states from the vacuum state $|0\rangle$. Indeed, they are symmetric in $\alpha \leftrightarrow \beta$ and will only generate states symmetric under the exchange of the two nodes. In order to generate the whole space of gauge invariant operators on the 2-node graph, we also need operators that act on a single node and change states without affecting neither the representations on the edges, nor the intertwiner state living at the other node. Natural candidates for such operators acting on $\mathcal{H}_{j_{1}, \ldots, j_{N}}^{(\alpha)}$ are the $E_{i j}^{(\alpha)} E_{j i}^{(\alpha)}$. They change the intertwiner living at the node $\alpha$ without changing the intertwiner living at $\beta$. Combining such local operators $E_{i}^{(\alpha)}, E_{i j}^{(\alpha)} E_{j i}^{(\alpha)}$ and $E_{i}^{(\beta)}$, $E_{i j}^{(\beta)} E_{j i}^{(\beta)}$ with the coupled symmetric operators $e_{i j}, f_{i j}, f_{i j}^{\dagger}$ allow to go between any two states in the Hilbert space ${ }^{2} \mathcal{H}$ and thus generate all gauge invariant operators on the 2-node graph.

\subsection{Symmetry reduction and cosmological analogies}

We now consider a symmetry reduction in our simple model that yields a homogeneous and isotropic sector. More specifically, we look for states in the Hilbert space ${ }^{2} \mathcal{H}$ invariant under a "global" $\mathrm{U}(N)$ symmetry generated by a generalization of the matching conditions $\mathcal{E}_{k}$, and which takes into account operators $E_{i j}$ acting on both nodes of the graph. Let us explain here the construction of the generators of this new $\mathrm{U}(N)$ symmetry and its action.

The matching conditions $\mathcal{E}_{k}$ break the $\mathrm{U}(N)$-actions on both nodes $\alpha$ and $\beta$. Nevertheless, we can see that the $\mathcal{E}_{k}$ generate a $\mathrm{U}(1)^{N}$ symmetry and that they are part of a larger $\mathrm{U}(N)$ symmetry algebra. We introduce the operators

$$
\mathcal{E}_{i j} \equiv E_{i j}^{(\alpha)}-E_{j i}^{(\beta)}=E_{i j}^{(\alpha)}-\left(E_{i j}^{(\beta)}\right)^{\dagger} .
$$

It is straightforward to compute their commutation relations and check that these operators form a $\mathfrak{u}(N)$ algebra

$$
\left[\mathcal{E}_{i j}, \mathcal{E}_{k l}\right]=\delta_{j k} \mathcal{E}_{i l}-\delta_{i l} \mathcal{E}_{k j}
$$

The diagonal operators are exactly the matching conditions $\mathcal{E}_{k k}=\mathcal{E}_{k}$ and generate the Cartan Abelian subalgebra of $\mathfrak{u}(N)$.

These $\mathcal{E}_{i j}$ 's generate $\mathrm{U}(N)$ transformations on the two intertwiner system. By construction, they act in $\mathcal{H}_{\otimes 2}$ as $(U, \bar{U})$ with the transformation on $\beta$ being the complex conjugate of the transformation on $\alpha$.

Two remarks are in order when computing the commutator between the matching condition and these new $\mathfrak{u}(N)$ generators,

$$
\left[\mathcal{E}_{i j}, \mathcal{E}_{k}\right]=\delta_{j k} \mathcal{E}_{i k}-\delta_{i k} \mathcal{E}_{k j}=\left(\delta_{j k}-\delta_{i k}\right) \mathcal{E}_{i j}
$$

On the one hand, we notice that the operators $\mathcal{E}_{i j}$ are not fully compatible with the matching conditions and they do not act on the 2-node Hilbert space ${ }^{2} \mathcal{H}$. Thus they do not generate a nontrivial $\mathrm{U}(N)$-action on ${ }^{2} \mathcal{H}$. On the other hand, we can look for vectors in ${ }^{2} \mathcal{H}$ which are invariant under this $\mathrm{U}(N)$ action, $\mathcal{E}_{i j}|\psi\rangle=0$ for all $i, j$. In particular, they will satisfy the matching conditions (given by the special case $i=j$ ). 
Following this line of thought, we introduce the subspace of spin network states which are invariant under this $\mathrm{U}(N)$-action

$$
{ }^{2} \mathcal{H}_{\mathrm{inv}} \equiv \operatorname{Inv}_{\mathrm{U}(N)}\left[{ }^{2} \mathcal{H}\right]=\operatorname{Inv}_{\mathrm{U}(N)}\left[\mathcal{H}_{\otimes 2}\right]=\operatorname{Inv}_{\mathrm{U}(N)}\left[\bigoplus_{J_{\alpha}, J_{\beta}} \mathcal{H}_{N}^{\left(J_{\alpha}\right)} \otimes \mathcal{H}_{N}^{\left(J_{\beta}\right)}\right] .
$$

Now taking into account that the spaces $\mathcal{H}_{N}^{(J)}$ are irreducible $\mathrm{U}(N)$-representations [53], requiring $\mathrm{U}(N)$-invariance imposes that the two representations for the two nodes are the same, $J_{\alpha}=J_{\beta}$, but furthermore there exists a unique invariant vector in the tensor product $\mathcal{H}_{N}^{(J)} \otimes \mathcal{H}_{N}^{(J)}$. We will call this unique invariant vector $|J\rangle$ and we will construct it explicitly in terms of the operators $e_{i j}$ and $f_{i j}$ in the next section.

It is important to notice that imposing $\mathrm{U}(N)$-invariance on our 2-node system, we obtain a single state $|J\rangle$ for every total boundary area $J$

$$
{ }^{2} \mathcal{H}_{\text {inv }}=\bigoplus_{J \in \mathbb{N}} \mathbb{C}|J\rangle
$$

We define these $|J\rangle$ states as the homogeneous and isotropic states of the model. The physical motivation behind this definition is that $\mathrm{U}(N)$ invariance is restricting our system to states which are not sensitive to area-preserving deformations of the boundary between $\alpha$ and $\beta$. They are isotropic in the sense that all directions (i.e. all links) are equivalent and the state only depends on the total boundary area, and they are homogeneous in the sense that the quantum state is the same at every point of space, i.e. at both nodes $\alpha$ and $\beta$ of our 2-node graph.

This allows to realize the reduction at the quantum level to the isotropic/homogeneous subspace by a straightforward $\mathrm{U}(N)$-group averaging. This opens the possibility of applying this logic to Loop Quantum Cosmology, which is based on a symmetry reduction at the classical level and a quantization $\grave{a}$ la loop of this reduced phase space. As it will be explained in the next section, the dynamics that we propose for the $\mathrm{U}(N)$ invariant sector has also strong analogies with the evolution operator used in LQC.

\subsection{Dynamics for the 2-node model}

In this section, we will define a consistent dynamics based on the $\mathrm{U}(N)$ invariance, restricting ourselves to the subspace of homogeneous/isotropic states described previously. In particular, such dynamics will automatically be consistent with the matching conditions.

\subsubsection{The algebra of $\mathrm{U}(N)$ invariant operators}

Before proposing a Hamiltonian operator for this system, we explore here the different $\mathrm{U}(N)$ invariant operators that we can construct. The most obvious one is the total boundary area operator $E$ itself. It is defined as $E=E^{(\alpha)}=E^{(\beta)}$ on the space ${ }^{2} \mathcal{H}$ of spin network states satisfying the matching condition. It is direct to check that it commutes with the $\mathfrak{u}(N)$ generators

$$
\left[\mathcal{E}_{i j}, E^{(\alpha)}\right]=\left[E_{i j}^{(\alpha)}, E^{(\alpha)}\right]=0=-\left[E_{j i}^{(\beta)}, E^{(\beta)}\right]=\left[\mathcal{E}_{i j}, E^{(\beta)}\right] .
$$

This total area operator is clearly diagonal in the basis $|J\rangle$,

$$
E|J\rangle=2 J|J\rangle
$$

Now, we need operators that could create dynamics on the space ${ }^{2} \mathcal{H}_{\text {inv }}$ by inducing transitions between states with different areas. To this purpose, we use the operators $e_{i j}, f_{i j}$ (equation (3.6)) 
and introduce the unique linear combinations that are $\mathrm{U}(N)$-invariant

$$
e \equiv \sum_{i j} e_{i j}=\sum_{i j} E_{i j}^{(\alpha)} E_{i j}^{(\beta)}, \quad f \equiv \sum_{i j} f_{i j}=\sum_{i j} F_{i j}^{(\alpha)} F_{i j}^{(\beta)}
$$

They obviously commute with the matching conditions since each operator $e_{i j}$ and $f_{i j}$ does. An important feature of these new operators, is that they are quadratic in $E$ and/or $F$ separately, so in this case their action is the same in both nodes. This actually ensure that the matching conditions hold.

It is convenient, for computational purposes, to introduce a shifted operator $\widetilde{e} \equiv e+2(E+$ $N-1)$. Then, using the quadratic constraints (3.3)-(3.5) satisfied by the operators $E_{i j}$ and $F_{i j}$, we can show that $\widetilde{e}, f$ and $f^{\dagger}$ form a simple algebra

$$
[\widetilde{e}, f]=-2(E+N+1) f, \quad\left[\widetilde{e}, f^{\dagger}\right]=2 f^{\dagger}(E+N+1), \quad\left[f, f^{\dagger}\right]=4(E+N) \widetilde{e}
$$

Written as such, it resembles to a $\mathfrak{s} l_{2}$ Lie algebra up to the factors in $E$, which is an operator and not a constant ${ }^{11}$.

We can use $f^{\dagger}$ as a creation operator. Thus we introduce the states

$$
|J\rangle_{\text {un }} \equiv f^{\dagger J}|0\rangle=\left(\sum_{i j} F_{i j}^{(\alpha) \dagger} F_{i j}^{(\beta) \dagger}\right)^{J}|0\rangle,
$$

where the index un stands for unnormalized. Since both the operator $f^{\dagger}$ and the vacuum state $|0\rangle$ are $\mathrm{U}(N)$-invariant, it is clear that the states $|J\rangle_{\text {un }}$ are also invariant under the $\mathrm{U}(N)$-action. Moreover, it is easy to check that they are eigenvectors of the total area operator:

$$
E|J\rangle_{\text {un }}=2 J|J\rangle_{\text {un }}
$$

so that they provide a basis for our Hilbert space ${ }^{2} \mathcal{H}_{\text {inv }}$ of homogeneous states. It is possible also to work with normalized states defined as

$$
|J\rangle \equiv \frac{1}{2^{J} J !(J+1) ! \sqrt{D_{N, J}}}|J\rangle_{\mathrm{un}},
$$

in terms of the dimension $D_{N, J}$ of the intertwiner space $\mathcal{H}_{N}^{(J)}$ [53] (see [32] for details).

The action of all $\widetilde{e}, f, f^{\dagger}$ operators over these normalized states is always quadratic in $J$ :

$$
\begin{aligned}
& \widetilde{e}|J\rangle=2(J+1)(N+J-1)|J\rangle, \\
& f|J\rangle=2 \sqrt{J(J+1)(N+J-1)(N+J-2)}|J-1\rangle, \\
& f^{\dagger}|J\rangle=2 \sqrt{(J+1)(J+2)(N+J)(N+J-1)}|J+1\rangle .
\end{aligned}
$$

From here, we see that it is possible to introduce renormalized operators that truly form a $\mathfrak{s} l_{2}$ algebra. We define the new operators:

$$
\begin{aligned}
& Z \equiv \frac{1}{\sqrt{E+2(N-1)}} \widetilde{e} \frac{1}{\sqrt{E+2(N-1)}}, \\
& X_{-} \equiv \frac{1}{\sqrt{E+2(N-1)}} f \frac{1}{\sqrt{E+2(N-1)}},
\end{aligned}
$$

\footnotetext{
${ }^{11}$ This is very similar to the $\mathfrak{s} l_{2}$ algebra $E_{\rho}^{(\alpha)}, F_{\bar{z}}^{(\alpha)}, F_{\bar{z}}^{(\alpha) \dagger}$ defined in [54] and used to build the $\mathrm{U}(N)$ coherent states.
} 


$$
X_{+} \equiv \frac{1}{\sqrt{E+2(N-1)}} f^{\dagger} \frac{1}{\sqrt{E+2(N-1)}} .
$$

Notice that the inverse square-root is well-defined since $E+2(N-1)$ is Hermitian and strictly positive as soon as $N \geq 2$. These operators are still $\mathrm{U}(N)$-invariant since $E$ is invariant too and we also have the Hermiticity relations, $Z^{\dagger}=Z$ and $X_{-}^{\dagger}=X_{+}$. Moreover, it is direct to compute the action of these renormalized operators on our $|J\rangle$ basis states.

To conclude, we can extract two important points

- The algebraic structure of the $\mathrm{U}(N)$-invariant space ${ }^{2} \mathcal{H}_{\text {inv }}$ of homogeneous states forms an irreducible unitary representation of $\mathfrak{s l}(2, \mathbb{R})$. The basis vectors $|J\rangle$ can be obtained by iterating the action of the creation/raising operator $f^{\dagger}$ (or $X_{+}$) on the vacuum state $|0\rangle$.

- This algebraic structure does not depend at all on the number of links $N$. Therefore, while working on homogeneous states, $N$ might have a physical meaning but it is not a relevant parameter mathematically. On the other hand, we expect it to become highly relevant when leaving the $\mathrm{U}(N)$-invariant subspace and studying inhomogeneities.

\subsubsection{The Hamiltonian}

In order to study the dynamics on this 2-node graph we propose the simplest $\mathrm{U}(N)$-invariant ansatz for a Hamiltonian operator

$$
H \equiv \eta \widetilde{e}+\left(\sigma f+\bar{\sigma} f^{\dagger}\right) .
$$

As explained above, the operator $\widetilde{e}$ does not affect the total boundary area, $[E, \widetilde{e}]=0$, while the operators $f$ and $f^{\dagger}$ respectively shrink and increase this area, $[E, f]=-2 f$ and $\left[E, f^{\dagger}\right]=+2 f^{\dagger}$. The coupling $\eta$ is real while $\sigma$ can be complex a priori, so that the operator $H$ is Hermitian. We can relate this Hamiltonian operation to the action of holonomy operators acting on all the loops of the 2-node graph [32]. From this point of view, our proposal is very similar to the standard ansatz for the quantum Hamiltonian constraint in LQG [103] and LQC (e.g. [10, 64]).

This Hamiltonian is quadratic in $J$ and we can give its explicit action on the basis states of ${ }^{2} \mathcal{H}_{\text {inv }}$ :

$$
\begin{aligned}
H|J\rangle= & \sigma 2 \sqrt{J(J+1)(N+J-1)(N+J-2)}|J-1\rangle+\eta 2(J+1)(N+J-1)|J\rangle \\
& +\bar{\sigma} 2 \sqrt{(J+1)(J+2)(N+J)(N+J-1)}|J+1\rangle,
\end{aligned}
$$

the details of the spectral properties of this Hamiltonian can be found in [32]. We observe in the spectral analysis that it presents three dynamical regimes depending on the value of the couplings that can be put in the same footing as the three regimes in LQC given by the sign of the cosmological constant [32]. Moreover, we can see that the structure of the Hamiltonian (3.7) is analogous to the one given in equation (2.11), with a reparametrization by a factor 2 of the label $J$. Notice that both labels $\mu$ and $J$ are area values, the coefficients of both equations are quadratic and both of them have contributions of three states labelled by $\mu, \mu-2$ and $\mu+2$ in the case of equation (2.11) and $J, J-1, J+1$ in the case of (3.7).

The ansatz given above is the most general $\mathrm{U}(N)$-invariant Hamiltonian (allowing only elementary changes in the total area), up to a renormalization by a $E$-dependent factor. Therefore, we can also propose renormalized Hamiltonian operators based on the renormalized operators considered in the previous section. For instance, we can define

$$
\mathbf{h} \equiv \frac{1}{\sqrt{E+2(N-1)}} H \frac{1}{\sqrt{E+2(N-1)}}=\eta Z+\left(\sigma X_{-}+\bar{\sigma} X_{+}\right) \in \mathfrak{s} l_{2} .
$$


We remark the fact that we study the action of these Hamiltonian operators, $H$ and $\mathbf{h}$, on the $\mathrm{U}(N)$-invariant space ${ }^{2} \mathcal{H}_{\text {inv }}$; nevertheless they are generally well-defined on the whole space of spinnetwork states ${ }^{2} \mathcal{H}$. Also, that the dynamics on the homogeneous sector does not depend mathematically on the parameter $N$ giving the number of links of the graph, as it does not appear in the action of the renormalized Hamiltonian $\mathbf{h}$.

The main characteristic of $\mathbf{h}$ is that it is an element in the Lie algebra $\mathfrak{s} l_{2}$ and its coefficients are linear in the variable $J$ at leading order. Thus we know its spectral properties from the representation theory of $\mathfrak{s} l_{2}$. The important point to underline here is that the three coupling regimes for the renormalized Hamiltonian $\mathbf{h}$ are exactly the same as for the original Hamiltonian $H$. This is very similar to the interplay between the evolution operator $\hat{\Theta}$ and the gravitational contribution to the Hamiltonian constraint $\hat{C}_{\text {grav }}$ (see e.g. [64]) in LQC. At the end of this section, we will obtain the classical counterpart of these Hamiltonians and we will be able to solve the equations of motion for $\mathbf{h}$ exactly.

\subsection{Classical setting: formulation of the 2-node model in terms of spinors}

One of the most interesting aspects of the $\mathrm{U}(N)$-framework is that it can be rewritten in terms of spinors rather straightforward [34,70]. This fact is natural in this setting because the operators in the $\mathrm{U}(N)$-formalism can be seen as the quantization of a classical spinorial model. This relationship may lead us to a better understanding of the geometrical meaning of the spinnetwork states in LQG and can also help us in the quest of a well defined semi-classical limit for the full theory.

Another remarkable point is the direct relation with the work by L. Freidel and S. Speziale so called "twisted geometries" $[55,56]$. Within this point of view, it was shown that the classical phase space of loop gravity on a given graph can be understood as a classical spinor model unravelling the connection between spinnetworks and the discrete geometry, mainly the Regge theory. This could be a key ingredient in order to shed light about the physical meaning of the spinfoam approach which treats the dynamics of the spinnetworks.

In this section we present the main elements allowing the recast of the $\mathrm{U}(N)$-framework in terms of spinors, showing how this is related with the usual SU(2) intertwiners in LQG. First of all, we define the classical spinor phase space. Later on, we propose a classical theory based on an action principle which actually gives us that phase space. After this classical step we perform the quantization, choosing a specific polarization based on certain spinorial holomorphic functionals and we will find that we obtain the correct intertwiner Hilbert space. Finally, we will discuss some interesting topics about the dynamics in our 2-node model using spinors and some points of contact with LQC.

\subsubsection{Spinors and notation}

In this part, we introduce the spinors and the related useful notations that we will be using in the rest of the section $[34,42,54,56,70]$. Given a spinor $z$

$$
|z\rangle=\left(\begin{array}{c}
z^{0} \\
z^{1}
\end{array}\right), \quad\langle z|=\left(\begin{array}{ll}
\bar{z}^{0} & \bar{z}^{1}
\end{array}\right)
$$

it is well known that there is a geometrical 3-vector $\vec{V}(z)$, defined from the projection of the $2 \times 2$ matrix $|z\rangle\langle z|$ onto Pauli matrices $\sigma_{a}$ (taken Hermitian and normalized so that $\left(\sigma_{a}\right)^{2}=\mathbb{I}$ )

$$
|z\rangle\langle z|=\frac{1}{2}(\langle z \mid z\rangle \mathbb{I}+\vec{V}(z) \cdot \vec{\sigma}) .
$$


It is straightforward to compute the norm and the components of this vector in terms of the spinors

$$
\begin{aligned}
& |\vec{V}(z)|=\langle z \mid z\rangle=\left|z^{0}\right|^{2}+\left|z^{1}\right|^{2}, \\
& V^{z}=\left|z^{0}\right|^{2}-\left|z^{1}\right|^{2}, \quad V^{x}=2 \operatorname{Re}\left(\bar{z}^{0} z^{1}\right), \quad V^{y}=2 \operatorname{Im}\left(\bar{z}^{0} z^{1}\right) .
\end{aligned}
$$

Also, it is important to notice that the spinor $z$ is entirely determined by the corresponding 3 -vector $\vec{V}(z)$ up to a global phase. We can give the reverse map

$$
z^{0}=e^{i \phi} \sqrt{\frac{|\vec{V}|+V^{z}}{2}}, \quad z^{1}=e^{i(\phi-\theta)} \sqrt{\frac{|\vec{V}|-V^{z}}{2}}, \quad \tan \theta=\frac{V^{y}}{V^{x}}
$$

where $e^{i \phi}$ is an arbitrary phase.

We can also introduce the map duality $\varsigma$ acting on spinors

$$
\varsigma\left(\begin{array}{c}
z^{0} \\
z^{1}
\end{array}\right)=\left(\begin{array}{c}
-\bar{z}^{1} \\
\bar{z}^{0}
\end{array}\right), \quad \varsigma^{2}=-1
$$

This is an anti-unitary map, $\langle\varsigma z \mid \varsigma w\rangle=\langle w \mid z\rangle=\overline{\langle z \mid w\rangle}$, and we will write the related state as

$$
\mid z] \equiv \varsigma|z\rangle, \quad[z \mid w]=\overline{\langle z \mid w\rangle} \text {. }
$$

This map $\varsigma$ maps the 3 -vector $\vec{V}(z)$ onto its opposite

$$
\mid z]\left[z \mid=\frac{1}{2}(\langle z \mid z\rangle \mathbb{I}-\vec{V}(z) \cdot \vec{\sigma}) .\right.
$$

Finally considering the setting necessary to describe intertwiners with $N$ legs, we consider $N$ spinors $z_{i}$ and their corresponding 3 -vectors $\vec{V}\left(z_{i}\right)$. Typically, we can require that the $N$ spinors satisfy a closure condition, i.e. that the sum of the corresponding 3 -vectors vanishes, $\sum_{i} \vec{V}\left(z_{i}\right)=0$. Coming back to the definition of the 3 -vectors $\vec{V}\left(z_{i}\right)$, the closure condition is easily translated in terms of $2 \times 2$ matrices

$$
\sum_{i}\left|z_{i}\right\rangle\left\langle z_{i}\right|=A(z) \mathbb{I}, \quad \text { with } \quad A(z) \equiv \frac{1}{2} \sum_{i}\left\langle z_{i} \mid z_{i}\right\rangle=\frac{1}{2} \sum_{i}\left|\vec{V}\left(z_{i}\right)\right| .
$$

This further translates into quadratic constraints on the spinors

$$
\sum_{i} z_{i}^{0} \bar{z}_{i}^{1}=0, \quad \sum_{i}\left|z_{i}^{0}\right|^{2}=\sum_{i}\left|z_{i}^{1}\right|^{2}=A(z)
$$

In simple terms, it means that the two components of the spinors, $z_{i}^{0}$ and $z_{i}^{1}$, are orthogonal $N$-vectors of equal norm. In order to simplify the notation, let us introduce the matrix elements of the $2 \times 2$ matrix $\sum_{i}\left|z_{i}\right\rangle\left\langle z_{i}\right|$

$$
\mathcal{C}_{a b}=\sum_{i} z_{i}^{a} \bar{z}_{i}^{b} .
$$

Then the unitary or closure conditions are written very simply

$$
\mathcal{C}_{00}-\mathcal{C}_{11}=0, \quad \mathcal{C}_{01}=\mathcal{C}_{10}=0
$$




\subsubsection{Phase space and quantization}

We are ready now to describe the phase space in terms of spinors. This will provide us with the suitable arena to proceed with the quantization [34, 70].

Then, we first introduce a simple Poisson bracket on our space of $N$ spinors

$$
\left\{z_{i}^{a}, \bar{z}_{j}^{b}\right\} \equiv i \delta^{a b} \delta_{i j}
$$

with all other brackets vanishing, $\left\{z_{i}^{a}, z_{j}^{b}\right\}=\left\{\bar{z}_{i}^{a}, \bar{z}_{j}^{b}\right\}=0$. This is exactly the Poisson bracket for $2 N$ decoupled harmonic oscillators.

We expect that the closure conditions generates global $\mathrm{SU}(2)$ transformations on the $N$ spinors. In order to check that, we have to compute the Poisson brackets between the various components of the $\mathcal{C}$-constraints:

$$
\begin{aligned}
& \left\{\mathcal{C}_{00}-\mathcal{C}_{11}, \mathcal{C}_{01}\right\}=-2 i \mathcal{C}_{01}, \quad\left\{\mathcal{C}_{00}-\mathcal{C}_{11}, \mathcal{C}_{10}\right\}=+2 i \mathcal{C}_{10}, \quad\left\{\mathcal{C}_{10}, \mathcal{C}_{01}\right\}=i\left(\mathcal{C}_{00}-\mathcal{C}_{11}\right), \\
& \left\{\operatorname{Tr} \mathcal{C}, \mathcal{C}_{00}-\mathcal{C}_{11}\right\}=\left\{\operatorname{Tr} \mathcal{C}, \mathcal{C}_{01}\right\}=\left\{\operatorname{Tr} \mathcal{C}, \mathcal{C}_{10}\right\}=0
\end{aligned}
$$

These four components $\mathcal{C}_{a b}$ do indeed form a closed $\mathfrak{u}(2)$ algebra with the three closure conditions $\mathcal{C}_{00}-\mathcal{C}_{11}, \mathcal{C}_{01}$ and $\mathcal{C}_{10}$ forming the $\mathfrak{s u}(2)$ subalgebra. Thus we will write $\overrightarrow{\mathcal{C}}$ for these three $\mathfrak{s} u(2)$ generators with $\mathcal{C}^{z} \equiv \mathcal{C}_{00}-\mathcal{C}_{11}$ and $\mathcal{C}^{+}=\mathcal{C}_{10}$ and $\mathcal{C}^{-}=\mathcal{C}_{01}$. The three closure conditions $\overrightarrow{\mathcal{C}}$ will actually become the generators $\vec{J}$ at the quantum level, while the operator $\operatorname{Tr} \mathcal{C}$ will correspond to the total energy/area $E$.

Now, let us define matrices $M$ and $Q$ in the following way

$$
\left.M_{i j}=\left\langle z_{i} \mid z_{j}\right\rangle=\overline{\left\langle z_{j} \mid z_{i}\right\rangle}, \quad Q_{i j}=\left\langle z_{j}\right| z_{i}\right]=\overline{\left[z_{i}\left|z_{j}\right\rangle\right.}=-\overline{\left[z_{j}\left|z_{i}\right\rangle\right.}
$$

with

$$
z_{i} \equiv\left(\begin{array}{l}
\bar{u}_{i 1} \sqrt{\lambda} \\
\bar{u}_{i 2} \sqrt{\lambda}
\end{array}\right), \quad \lambda \equiv \operatorname{Tr} M / 2
$$

and $u_{i j}$ elements of a unitary matrix. It is possible to write this matrices as

$$
M=\lambda U \Delta U^{-1}, \quad \Delta=\left(\begin{array}{lll}
1 & & \\
& 1 & \\
\hline & & 0_{N-2}
\end{array}\right), \quad Q=\lambda U \Delta_{\epsilon}{ }^{t} U, \quad \Delta_{\epsilon}=\left(\begin{array}{ccc}
1 & \\
-1 & & \\
\hline & & 0_{N-2}
\end{array}\right),
$$

where $U$ is a unitary matrix $U^{\dagger} U=\mathbb{I}$.

It is easy to show that indeed (up to a global phase) these matrices are the most general ones satisfying $M=M^{\dagger},{ }^{t} Q=-Q$ and the classical analogs to the quadratic constraints satisfied by the operators $E$ and $F$. On the other hand there is a fundamental point in this construction which is that the unitarity condition on the matrices $U$ is equivalent (with the presented definition of the spinors in terms of the unitary matrix elements) to the closure conditions on the spinors.

Now, we can also compute the Poisson brackets of the $M_{i j}$ and $Q_{i j}$ matrix elements:

$$
\begin{aligned}
& \left\{M_{i j}, M_{k l}\right\}=i\left(\delta_{k j} M_{i l}-\delta_{i l} M_{k j}\right), \quad\left\{M_{i j}, Q_{k l}\right\}=i\left(\delta_{j k} Q_{i l}-\delta_{j l} Q_{i k}\right), \\
& \left\{Q_{i j}, Q_{k l}\right\}=0, \quad\left\{\bar{Q}_{i j}, Q_{k l}\right\}=i\left(\delta_{i k} M_{l j}+\delta_{j l} M_{k i}-\delta_{j k} M_{l i}-\delta_{i l} M_{k j}\right),
\end{aligned}
$$

which reproduces the expected commutators (3.1) up to the $i$-factor. We further check that these variables commute with the closure constraints generating the $\mathrm{SU}(2)$ transformations

$$
\left\{\overrightarrow{\mathcal{C}}, M_{i j}\right\}=\left\{\overrightarrow{\mathcal{C}}, Q_{i j}\right\}=0
$$


Finally, we look at their commutator with $\operatorname{Tr} \mathcal{C}$

$$
\left\{\operatorname{Tr} \mathcal{C}, M_{i j}\right\}=0, \quad\left\{\operatorname{Tr} \mathcal{C}, Q_{i j}\right\}=\left\{\sum_{k} M_{k k}, Q_{i j}\right\}=+2 i Q_{i j}
$$

which confirms that the matrix $M$ is invariant under the full $\mathrm{U}(2)$ subgroup and that $\operatorname{Tr} \mathcal{C}$ acts as a dilatation operator on the $Q$ variables, or reversely that the $Q_{i j}$ acts as creation operators for the total energy/area variable $\operatorname{Tr} \mathcal{C}$.

So far, we have been able to characterize the classical phase space associated to the spinors $z_{i}$ and the variables $M_{i j}, Q_{i j}$. Then we can now proceed to the quantization. In order to do that, we introduce the Hilbert spaces $\mathcal{H}_{J}^{(Q)}$ of homogeneous polynomials in the $Q_{i j}$ of degree $J$

$$
\mathcal{H}_{J}^{(Q)} \equiv\left\{P \in \mathbb{P}\left[Q_{i j}\right] \mid P\left(\rho Q_{i j}\right)=\rho^{J} P\left(Q_{i j}\right), \forall \rho \in \mathbb{C}\right\} .
$$

These are polynomials completely anti-holomorphic in the spinors $z_{i}$ and of order $2 J$.

One can prove that these Hilbert spaces $\mathcal{H}_{J}^{(Q)}$ are isomorphic to the Hilbert space $\mathcal{H}_{N}^{(J)}$ of $N$-valent intertwiners with fixed total area $J$. To this purpose, we will construct the explicit representation of the operators quantizing $M_{i j}$ and $Q_{i j}$ on the spaces $\mathcal{H}_{J}^{(Q)}$ and show that they match the actions of the $\mathrm{U}(N)$ operators $E_{i j}$ and $F_{i j}^{\dagger}$ which we described earlier. Our quantization relies on quantizing the $\bar{z}_{i}$ as multiplication operators while promoting $z_{i}$ to a derivative operator

$$
\widehat{\bar{z}}_{i}^{a} \equiv \bar{z}_{i}^{a} \times, \quad \widehat{z}_{i}^{a} \equiv \frac{\partial}{\partial \bar{z}_{i}^{a}},
$$

which satisfies the commutator $[\hat{z}, \hat{\bar{z}}]=1$ as expected for the quantization of the classical bracket $\{z, \bar{z}\}=i$. Then, we quantize the matrix elements $M_{i j}$ and $Q_{i j}$ and the closure constraints following this correspondence:

$$
\begin{array}{ll}
\widehat{M}_{i j}=\bar{z}_{i}^{0} \frac{\partial}{\partial \bar{z}_{j}^{0}}+\bar{z}_{i}^{1} \frac{\partial}{\partial \bar{z}_{j}^{1}}, & \widehat{Q}_{i j}=\bar{z}_{i}^{0} \bar{z}_{j}^{1}-\bar{z}_{i}^{1} \bar{z}_{j}^{0}=Q_{i j}, \\
\widehat{\bar{Q}}_{i j}=\frac{\partial^{2}}{\partial \bar{z}_{i}^{0} \partial \bar{z}_{j}^{1}}-\frac{\partial^{2}}{\partial \bar{z}_{i}^{1} \partial \bar{z}_{j}^{0}}, & \widehat{\mathcal{C}}_{a b}=\sum_{k} \bar{z}_{k}^{b} \frac{\partial}{\partial \bar{z}_{k}^{a}} .
\end{array}
$$

It is straightforward to check that the $\widehat{\mathcal{C}}_{a b}$ and the $\widehat{M}_{i j}$ respectively form a $\mathfrak{u}(2)$ and a $\mathfrak{u}(N)$ Lie algebra, as expected

$$
\left[\widehat{\mathcal{C}}_{a b}, \widehat{\mathcal{C}}_{c d}\right]=\delta_{a d} \widehat{\mathcal{C}}_{c b}-\delta_{c b} \widehat{\mathcal{C}}_{a d}, \quad\left[\widehat{M}_{i j}, \widehat{M}_{k l}\right]=\delta_{k j} \widehat{M}_{i l}-\delta_{i l} \widehat{M}_{k j}, \quad\left[\widehat{\mathcal{C}}_{a b}, \widehat{M}_{i j}\right]=0,
$$

which amounts to multiply the Poisson bracket (3.8) and (3.9) by $-i$. Then, we first check the action of the closure constraints on functions of the variables $Q_{i j}$ :

$$
\begin{aligned}
& \widehat{\overrightarrow{\mathcal{C}}} Q_{i j}=0, \quad \widehat{(\operatorname{Tr} \mathcal{C})} Q_{i j}=2 Q_{i j}, \\
& \forall P \in \mathcal{H}_{J}^{(Q)}=\mathbb{P}_{J}\left[Q_{i j}\right], \quad \widehat{\overrightarrow{\mathcal{C}}} P\left(Q_{i j}\right)=0, \quad \widehat{(\operatorname{Tr} \mathcal{C})} P\left(Q_{i j}\right)=2 J P\left(Q_{i j}\right),
\end{aligned}
$$

so that our wavefunctions $P \in \mathcal{H}_{J}^{(Q)}$ are $\mathrm{SU}(2)$-invariant (vanish under the closure constraints) and are eigenvectors of the $\operatorname{Tr} \mathcal{C}$-operator with eigenvalue $2 J$.

Second, we check that the operators $\widehat{M}$ and $\widehat{(\operatorname{Tr} \mathcal{C})}$ satisfy the same quadratic constraints on the Hilbert space $\mathcal{H}_{J}^{(Q)}$ (i.e. assuming that the operators acts on $\mathrm{SU}(2)$-invariant functions vanishing under the closure constraints) that the $\mathfrak{u}(N)$-generators $E_{i j}$

$$
\widehat{(\operatorname{Tr} \mathcal{C})}=\sum_{k} \widehat{M}_{k k}, \quad \sum_{k} \widehat{M}_{i k} \widehat{M}_{k j}=\widehat{M}_{i j}\left(\frac{\widehat{(\operatorname{Tr} \mathcal{C})}}{2}+N-2\right),
$$


which allows us to get the value of the (quadratic) $\mathrm{U}(N)$-Casimir operator on the space $\mathcal{H}_{J}^{(Q)}$ :

$$
\sum_{i k} \widehat{M}_{i k} \widehat{M}_{k i}=\widehat{(\operatorname{Tr} \mathcal{C})}\left(\frac{\widehat{(\operatorname{Tr} \mathcal{C})}}{2}+N-2\right)=2 J(J+N-2)
$$

Thus, we can safely conclude that this provides a proper quantization of our spinors and $M$ variables, which matches exactly with the $\mathfrak{u}(N)$-structure on the intertwiner space (with the exact same ordering)

$$
\mathcal{H}_{J}^{(Q)} \sim \mathcal{H}_{N}^{(J)}, \quad \widehat{M}_{i j}=E_{i j}, \quad \widehat{(\operatorname{Tr} \mathcal{C})}=E
$$

Now, turning to the $\widehat{Q}_{i j}$-operators, it is straightforward to check that they have the exact same action that the $F_{i j}^{\dagger}$ operators, they satisfy the same Lie algebra commutators (3.1) and the same quadratic constraints (3.3)-(3.5). Clearly, the simple multiplicative action of an operator $\widehat{Q}_{i j}$ send a polynomial in $\mathbb{P}_{J}\left[Q_{i j}\right]$ to a polynomial in $\mathbb{P}_{J+1}\left[Q_{i j}\right]$. Reciprocally, the derivative action of $\widehat{\bar{Q}}_{i j}$ decreases the degree of the polynomials and maps $\mathbb{P}_{J+1}\left[Q_{i j}\right]$ onto $\mathbb{P}_{J}\left[Q_{i j}\right]$.

Finally, let us look at the scalar product on the whole space of polynomials $\mathbb{P}\left[Q_{i j}\right]$. In order to ensure the correct Hermiticity relations for $\widehat{M}_{i j}$ and $\widehat{Q}_{i j}, \widehat{\bar{Q}}_{i j}$, it seems that we have a unique measure (up to a global factor)

$$
\forall \phi, \psi \in \mathbb{P}\left[Q_{i j}\right], \quad\langle\phi \mid \psi\rangle \equiv \int \prod_{i} d^{4} z_{i} e^{-\sum_{i}\left\langle z_{i} \mid z_{i}\right\rangle} \overline{\phi\left(Q_{i j}\right)} \psi\left(Q_{i j}\right)
$$

Then it is easy to check that we have $\widehat{M}_{i j}^{\dagger}=\widehat{M}_{j i}$ and $\widehat{Q}_{i j}^{\dagger}=\widehat{\bar{Q}}_{i j}$ as wanted.

It is easy to see that the spaces of homogeneous polynomials $\mathbb{P}_{J}\left[Q_{i j}\right]$ are orthogonal with respect to this scalar product. The quickest way to realize that this is true is to consider the operator $\widehat{(\operatorname{Tr} \mathcal{C})}$, which is Hermitian with respect to this scalar product and takes different values on the spaces $\mathbb{P}_{J}\left[Q_{i j}\right]$ depending on the value of $J$. Thus these spaces $\mathbb{P}_{J}\left[Q_{i j}\right]$ are orthogonal to each other.

This concludes our quantization procedure thus showing that the intertwiner space for $N$ legs and fixed total area $J=\sum_{i} j_{i}$ can be seen as the space of homogeneous polynomials in the $Q_{i j}$ variables with degree $J$. This provides us with a description of the intertwiners as wave-functions anti-holomorphic in the spinors $z_{i}$ constrained by the closure conditions ${ }^{12}$.

\subsubsection{Action principle}

It is possible to write an action principle for the previous Poisson bracket structure in terms of the spinors. In order to be consistent, we have to take into account the closure constraint, but also the matching conditions coming from the gluing of several intertwiners together. Furthermore, we propose an interaction term (a Hamiltonian) for this model.

Once we know that the Hilbert space of LQG can be described as the quantization of the phase space in terms of spinors (with the construction explained above) [70], it is interesting to present explicitly the correspondence between the standard formalism of loop (quantum) gravity and the spinor formulation provided by the reconstruction of the $\mathrm{SU}(2)$ group element $g_{\ell}$ in terms of the spinors [56].

\footnotetext{
${ }^{12}$ It is also possible to present an alternative construction [34], which can be considered as "dual" to the representation defined above. It is based on the coherent states for the oscillators, thus recovering the framework of the $\mathrm{U}(N)$ coherent intertwiner states introduced in [54] and further developed in [42].
} 
Considering an link $\ell$ with the two spinors at each of its end-nodes $z_{s(\ell), \ell}$ and $z_{t(\ell), \ell}$, there exists a unique $\mathrm{SU}(2)$ group element mapping one onto the other. More precisely

$$
g_{\ell} \equiv \frac{\left.\mid z_{s(\ell), \ell}\right]\left\langle z_{t(\ell), \ell}|-| z_{s(\ell), \ell}\right\rangle\left[z_{t(\ell), \ell} \mid\right.}{\sqrt{\left\langle z_{s(\ell), \ell} \mid z_{s(\ell), \ell}\right\rangle\left\langle z_{t(\ell), \ell} \mid z_{t(\ell), \ell}\right\rangle}}
$$

is uniquely fixed by the following conditions

$$
\begin{aligned}
& g_{\ell} \frac{\left|z_{t(\ell), \ell}\right\rangle}{\sqrt{\left\langle z_{t(\ell), \ell} \mid z_{t(\ell), \ell}\right\rangle}}=\frac{\left.\mid z_{s(\ell), \ell}\right]}{\sqrt{\left\langle z_{s(\ell), \ell} \mid z_{s(\ell), \ell}\right\rangle}}, \\
& g_{\ell} \frac{\left.\mid z_{t(\ell), \ell}\right]}{\sqrt{\left\langle z_{t(\ell), \ell} \mid z_{t(\ell), \ell}\right\rangle}}=-\frac{\left|z_{s(\ell), \ell}\right\rangle}{\sqrt{\left\langle z_{s(\ell), \ell} \mid z_{s(\ell), \ell}\right\rangle}}, \quad g_{\ell} \in \mathrm{SU}(2),
\end{aligned}
$$

thus sending the source normalized spinor onto the dual of the target normalized spinor.

Starting from this point, it is possible to construct objects in terms of the matrices $M$ and $Q$ that are $\mathrm{SU}(2)$ invariant and satisfying the matching conditions [34]. The expression of these objets (dubbed as "generalized holonomies" for their close relation with the usual holonomy operators in LQG) is

$$
\begin{aligned}
\mathcal{M}_{\mathcal{L}}^{\left\{r_{i}\right\}} \equiv & \prod_{i} r_{i-1} r_{i} \bar{Q}_{i, i-1}^{i}+\left(1-r_{i-1}\right) r_{i} M_{i-1, i}^{i}+r_{i-1}\left(1-r_{i}\right) M_{i, i-1}^{i} \\
& +\left(1-r_{i-1}\right)\left(1-r_{i}\right) Q_{i, i-1}^{i}=\prod_{i}\left\langle\varsigma^{r_{i-1}} z_{v_{i}, e_{i-1}} \mid \varsigma^{1-r_{i}} z_{v_{i}, e_{i}}\right\rangle
\end{aligned}
$$

with $r_{i}=0,1$; and $M_{j, k}^{v}, Q_{j, k}^{v}$ the corresponding operators for the node $v$ of a given loop of a generic graph $\Gamma$ (Fig. 6) and acting on the links $j$ and $k$. These objects will be the building blocks for the interaction term of our model.

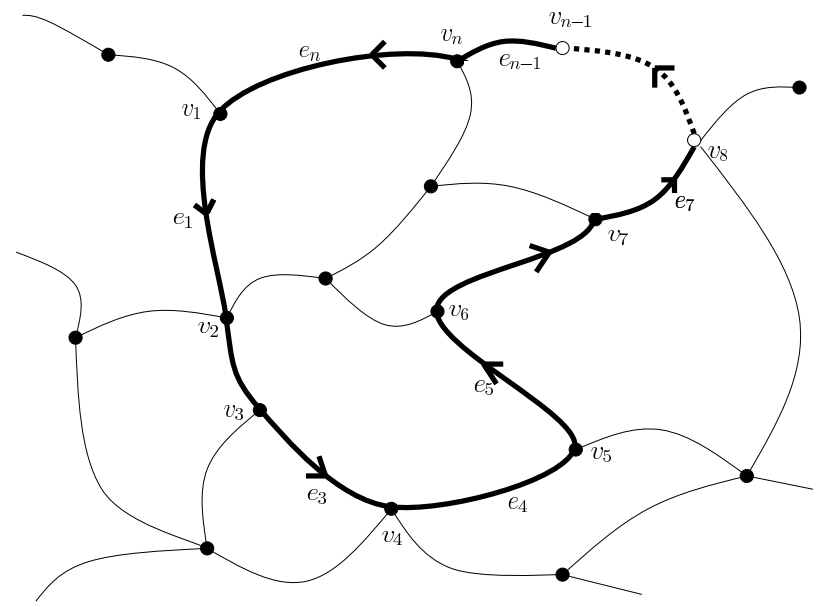

Figure 6. The loop $\mathcal{L}=\left\{\ell_{1}, \ell_{2}, \ldots, \ell_{n}\right\}$ on the graph $\Gamma$.

Using all these ingredients, we want to write an action principle for this formalism. We should keep in mind that the spinnetwork states on a given graph $\Gamma$ are $V$ intertwiner states - one at each node $v$-glued together along the links $e$ so that they satisfy the matching conditions on each link. Consequently the phase space consists with the spinors $z_{v, \ell}$ (where $e$ are links attached to the node $v$, i.e. such that $v=s(\ell)$ or $v=t(\ell))$ which we constrain by the closure conditions $\overrightarrow{\mathcal{C}^{v}}$ at each node $v$ and the matching conditions on each link $e$. The corresponding 
action reads

$$
\begin{aligned}
S_{0}^{\Gamma}\left[z_{v, \ell}\right]= & \int d t \sum_{v} \sum_{e \mid v \in \partial e}\left(-i\left\langle z_{v, \ell} \mid \partial_{n} z_{v, \ell}\right\rangle+\left\langle z_{v, \ell}\left|\Lambda_{v}\right| z_{v, \ell}\right\rangle\right) \\
& +\sum_{\ell} \rho_{\ell}\left(\left\langle z_{s(\ell), \ell} \mid z_{s(\ell), \ell}\right\rangle-\left\langle z_{t(\ell), \ell} \mid z_{t(\ell), \ell}\right\rangle\right)
\end{aligned}
$$

where the $2 \times 2$ Lagrange multipliers $\Lambda_{v}$ satisfying $\operatorname{Tr} \Lambda_{v}=0$ impose the closure constraints and the Lagrange multipliers $\rho_{\ell} \in \mathbb{R}$ impose the matching conditions. All the constraints are first class, they generate $\mathrm{SU}(2)$ transformations at each node and $\mathrm{U}(1)$ transformations on each link $e$.

We can analogously describe this system parameterized by $N_{v} \times N_{v}$ unitary matrices $U^{v}$ and the parameters $\lambda_{v}$. The matrix elements $U_{e f}^{v}$ refer to pairs of links $e, f$ attached to the node $v$. As it was mention before, the closure conditions are automatically encoded in the requirement that the matrices $U^{v}$ are unitary. Of course, we still have to impose the matching conditions $M_{e e}^{s(\ell)}-M_{e e}^{t(\ell)}=0$ on each link $e$ where the matrices $M^{v}=\lambda_{v} U^{v} \Delta U^{v-1}$ are functions of both $\lambda_{v}$ and $U^{v}$. So in this case, the action ${ }^{13}$ reads

$$
S_{0}^{\Gamma}\left[\lambda_{v}, U^{v}\right]=\int d t \sum_{v}\left(-i \lambda_{v} \operatorname{Tr} U^{v} \Delta \partial_{n} U^{v \dagger}-\operatorname{Tr} \Theta_{v}\left(U^{v} U^{v \dagger}-\mathbb{I}\right)\right)+\sum_{\ell} \rho_{\ell}\left(M_{e e}^{s(\ell)}-M_{e e}^{t(\ell)}\right),
$$

where the $\rho_{\ell}$ impose the matching conditions while the $N_{v} \times N_{v}$ matrices $\Theta_{v}$ are the Lagrange multipliers for the unitarity of the matrices $U^{v}$.

This free action describes the classical kinematics of spinnetworks on the graph $\Gamma$. Now, we are going to add interaction terms to this action. Such interaction terms are built with the generalized holonomy observables $\mathcal{M}_{\mathcal{L}}^{\left\{r_{i}\right\}}$. With this construction, the closure and matching conditions are trivially satisfied. Our proposal for a classical action for spinnetworks with nontrivial dynamics is thus

$$
S_{\left.\gamma_{\mathcal{L}} \Gamma_{i}\right\}}=S_{0}^{\Gamma}+\int d t \sum_{\mathcal{L},\left\{r_{i}\right\}} \gamma_{\mathcal{L}}^{\left\{r_{i}\right\}} \mathcal{M}_{\mathcal{L}}^{\left\{r_{i}\right\}}
$$

where the $\gamma_{\mathcal{L}}^{\left\{r_{i}\right\}}$ are the coupling constants giving the relative weight of each generalized holonomy in the full Hamiltonian. We will study in more detail this classical action principle in the specific case of the 2-node graph in the following section.

\subsubsection{Effective dynamics for the 2-node graph}

Let us particularize to the 2-node graph the action principle proposed before for a general graph. Then, the action for this model, including a general interaction term is

$$
S\left[U^{\alpha}, U^{\beta}, \lambda\right] \equiv S_{0}\left[U^{\alpha}, U^{\beta}, \lambda\right]+\int d t \sum_{i, j}\left[\gamma_{i j}^{+} Q_{i j}^{\alpha} Q_{i j}^{\beta}+\gamma_{i j}^{-} \bar{Q}_{i j}^{\alpha} \bar{Q}_{i j}^{\beta}+\gamma_{i j}^{0} M_{i j}^{\alpha} M_{i j}^{\beta}\right]
$$

with

$$
S_{0}\left[U^{\alpha}, U^{\beta}, \lambda\right] \equiv \int d t\left(-i \lambda\left[\operatorname{Tr} U^{\alpha} \Delta \partial_{n} U^{\alpha \dagger}+\operatorname{Tr} U^{\beta} \Delta \partial_{n} U^{\beta \dagger}\right]\right.
$$

\footnotetext{
${ }^{13}$ This action is invariant under the action of $\mathrm{SU}(2) \times \mathrm{U}\left(N_{v}-2\right)$ at every node, which reduces the number of degrees of freedom of the matrices $U^{v}$ to the spinors $z_{v, \ell}$ which are actually the two first columns of those matrices.
} 


$$
\left.+\sum_{i} \rho_{i}\left[\left(U^{\alpha} \Delta U^{\alpha \dagger}\right)_{i i}-\left(U^{\beta} \Delta U^{\beta \dagger}\right)_{i i}\right]\right)
$$

where $\lambda \equiv \lambda_{\alpha}=\lambda_{\beta}$, due to the matching conditions, and the $\gamma$ 's are coupling constants satisfying

$$
\gamma^{-}=\overline{\gamma^{+}}, \quad \gamma^{0}=\left(\gamma^{0}\right)^{\dagger}
$$

in order to have a real Hamiltonian.

At this point one can look for the classical counterpart of the quantum Hamiltonian for the homogeneous and isotropic sector imposing a global $\mathrm{U}(N)$ symmetry. After this, the action depends just on two conjugated variables $\lambda \equiv \operatorname{Tr} M / 2$ and $\phi$. Due to the symmetry reduction and the matching conditions, $\phi$ relates the unitary matrix (or spinor) in the node $\alpha$ with the one at $\beta: U^{\alpha}=e^{i \phi} \overline{U^{\beta}}$. Finally, the expression for the action for the reduced sector is

$$
S_{\mathrm{inv}}[\lambda, \phi]=-2 \int d t\left(\lambda \partial_{n} \phi-\lambda^{2}\left(\gamma^{0}-\gamma^{+} e^{2 i \phi}-\gamma^{-} e^{-2 i \phi}\right)\right),
$$

with the Hamiltonian $H=\lambda^{2}\left(\gamma^{0}-2 \gamma \cos (2 \phi)\right)$.

This Hamiltonian corresponds with the quantum Hamiltonian $H$ that we have considered before. As we did also there, we can introduce the renormalized Hamiltonian

$$
\mathbf{h} \equiv \frac{1}{\lambda} H=\lambda\left(\gamma^{0}-2 \gamma \cos (2 \phi)\right)
$$

that is still $\mathrm{SU}(2)$ and $\mathrm{U}(N)$ invariant.

The equations of motion coming from the new Hamiltonian $\mathbf{h}$ are simply given by

$$
\partial_{n} \phi=\gamma^{0}-2 \gamma \cos (2 \phi), \quad \partial_{n} \lambda=-4 \gamma \lambda \sin (2 \phi) .
$$

We can solve exactly these differential equations. First we solve for $\phi(t)$ analytically and then the following expression for $\lambda$ in terms of $\phi$ solves the equations of motions

$$
\lambda=\frac{\epsilon}{\gamma^{0}-2 \gamma \cos (2 \phi)},
$$

where $\epsilon= \pm$ is a global sign. Let us point out that the equation of motion for $\lambda$ only determines it up a global numerical factor. Then we should remember that $\lambda$ is the total area and we always constrain it to be positive. Moreover this is the equation of a conic with radial coordinate given by $\lambda$, polar coordinate $2 \phi$ and eccentricity $2 \gamma / \gamma_{0}$.

The solutions ${ }^{14}$ for $\phi(t)$, depending on the different values for the parameters $\gamma^{0}$ and $\gamma$ are elliptic region $\left(\left|\gamma^{0}\right|>2|\gamma|\right)$ :

$$
\phi(t)=-\arctan \left(\frac{\left(2 \gamma-\gamma^{0}\right) \tan \left(t \sqrt{\left(\gamma^{0}\right)^{2}-4 \gamma^{2}}\right)}{\sqrt{\left(\gamma^{0}\right)^{2}-4 \gamma^{2}}}\right),
$$

hyperbolic region $\left(\left|\gamma^{0}\right|<2|\gamma|\right)$ :

$$
\phi(t)=-\arctan \left(\frac{\sqrt{4 \gamma^{2}-\left(\gamma^{0}\right)^{2}}}{\left(2 \gamma+\gamma^{0}\right) \tanh \left(t \sqrt{4 \gamma^{2}-\left(\gamma^{0}\right)^{2}}\right)}\right),
$$

\footnotetext{
${ }^{14}$ We have chosen the most convenient constants of integration due to the fact that this constants are just translations in the temporal variable
} 
parabolic region $\mathbf{I}\left(\gamma^{0}=2 \gamma\right)$ :

$$
\phi(t)=-\arctan \left(\frac{1}{4 \gamma t}\right)
$$

parabolic region II $\left(\gamma^{0}=-2 \gamma\right)$ :

$$
\phi(t)=-\arctan (4 \gamma t) .
$$

Let us give a brief description of these solutions (Fig. 7). First, we notice that we get the same solution $\lambda(t)$ for the two cases I and II of the parabolic region by taking $\epsilon=+$ in case I and $\epsilon=-$ in case II. In the elliptic case, we have a system in which the area $\lambda$ has an oscillatory behavior. While in the other two regimes the area shrinks under evolution, reaches a minimum value and then increases until infinity. As it was pointed out in [32, 34], the quantum Hamiltonian of this 2-node model is mathematically analogous to the gravitational part of the Hamiltonian in LQC. Following this analogy, we can interpret the results obtained here as the classical analogous of the quantum big bounce found in LQC.

At this point, it would be very interesting to go beyond the $\mathrm{U}(N)$-invariant sector. The action (3.10) defines the full classical kinematics and dynamics of spin network states on the 2node graph. It is a nontrivial matrix model defined in terms of the unitary matrices $U^{\alpha}$ and $U^{\beta}$ and with quartic interaction terms. Even if we still choose a $\mathrm{U}(N)$-invariant Hamiltonian of the the type $\gamma^{+} \operatorname{Tr} Q^{\alpha} Q^{\beta}+\gamma^{-} \operatorname{Tr} \bar{Q}^{\alpha} \bar{Q}^{\beta}+\gamma^{0} \operatorname{Tr} M^{\alpha t} M^{\beta}$, this will nevertheless induce nontrivial dynamics for the matrices $U^{\alpha}$ and $U^{\beta}$. It would be very interesting to follow this line of research and study what kind of anisotropy does this model describe in the context of loop cosmology.

Finally, let us point out some other possible lines of research on these topics. Although the results on the 2-node graph are compelling due to the relation with cosmology, there are some limitations. Up to now, it has not been possible to go beyond the homogeneous sector. The relation with the improved dynamics in LQC and the introduction of matter are still missing. It would be interesting to generalize the methods presented here to more complicated graphs in order to test the truncation of LQG to a fixed graph. We point out two such generalizations. One is the work with the graph with $3+N$ nodes in [32]. This graph could allow us, for instance, to study rotations or black-hole radiation processes. An interesting generalization could be to consider a graph with an infinite number of nodes in order to study a continuum limit. These models can be generalized in numerous directions and we think that they can contribute to the understanding of the framework and shed light on fundamental problems in LQG and spinfoams.

\section{The covariant few-node model}

So far we have presented the use of the 2-nodes graph in the canonical theory, seeing how this can be used as a truncation of the full theory to a finite number of degrees of freedom, and we have seen different techniques to impose homogeneity and isotropy on this system in order to obtain the description of a FLRW universe. On the other hand, in our description we can not break free from the ambiguities in the definition of the dynamics: there is no agreement about the form of the Hamiltonian constraints used. We hope that the works presented in this review could add new insights to this question.

The dynamics of Loop Quantum Gravity, however, admits also a covariant formulation in terms of transition amplitudes, which appears to be far less subject to ambiguities. The fundamental object is a general covariant path integral, interpreted as a sum over geometries. In this section we review calculation of the transition amplitude between homogeneous isotropic states on a regular graph. This calculation was first performed in the context of the 2-node model [31] and extended to the case with a cosmological constant in [28], but was later extended 

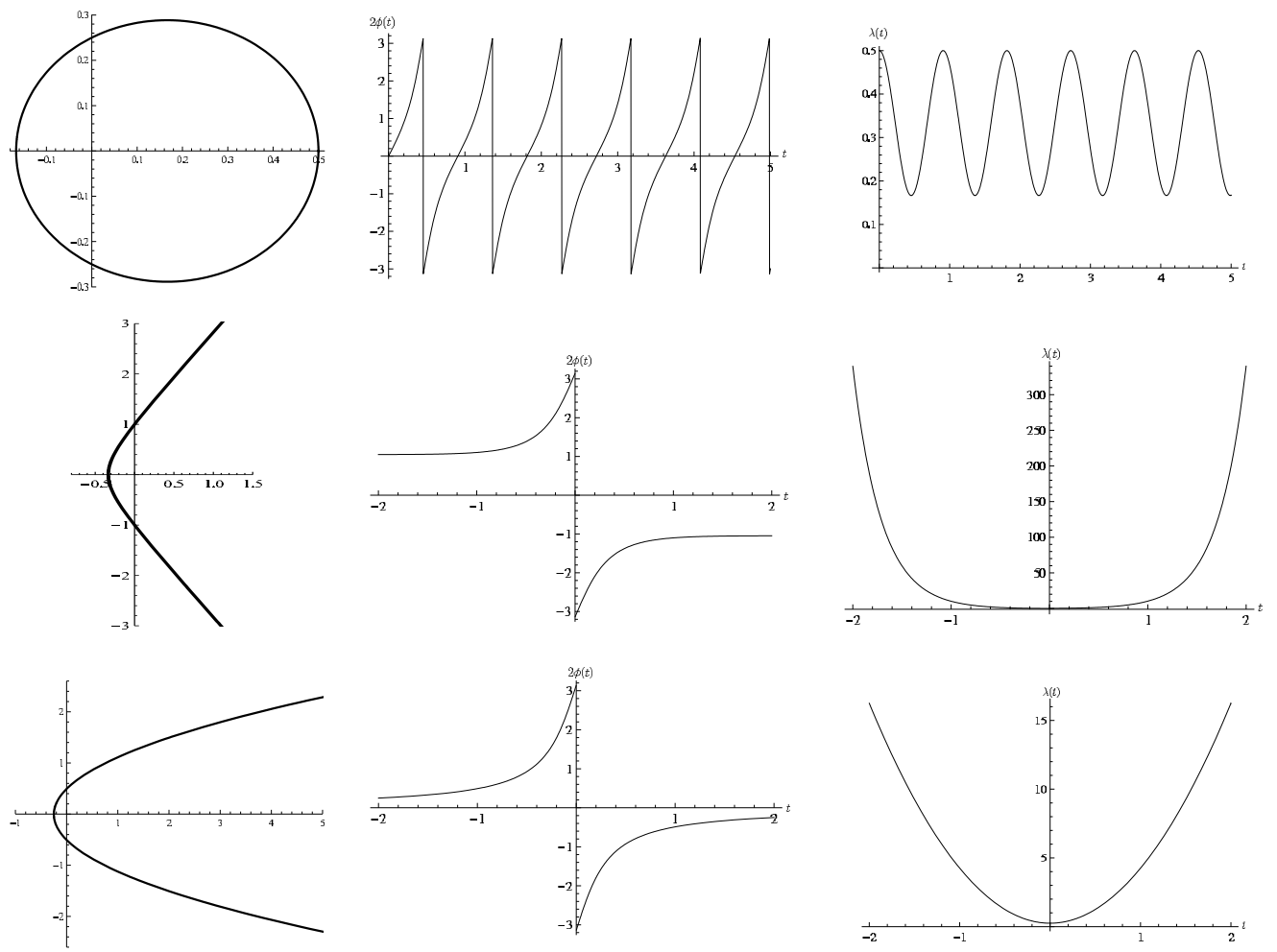

Figure 7. We plot the behavior of $\phi(t)$ and $\lambda(t)$ (given by the equations (3.11) and (3.12)) in the three different regimes for $\gamma=1$ and respectively $\gamma^{0}=4$ (elliptic regime), $\gamma^{0}=1$ (hyperbolic regime) and finally $\gamma^{0}=2$ (parabolic regime). In the first column, we give the polar plots constructed by taking as polar coordinates $(2 \phi, \lambda(\phi))$. The second column gives for $\phi(t)$ and the third one $\lambda(t)$. We observe in those plots the periodical behavior of $\lambda$ (interpreted as the total area of the model) as a function of time in the elliptic case and a behavior analogous to a cosmological big bounce in the other two cases.

to an arbitrary regular graph in [107]. Here we give directly the general case with a cosmological constant on an arbitrary regular graph.

\subsection{Brief introduction to spinfoam theory}

The transition amplitudes are obtained by summing over all the possible spinfoams. A spinfoam is a 4-dimensional simplicial 2-complex $\mathcal{C}$ colored with spins $j_{f}$ and intertwiners $i_{e}$, associated, respectively, to the faces $f$ and the edges $e$, i.e. the 3 -cells. This is the object that encodes the quantum geometry (Penrose's spin-geometry theorem). Here we take the sum over the coloring $j_{f}$ and $i_{\ell}$, the product of "face amplitude" $\prod_{f} d\left(j_{f}\right)$ and a product of vertex amplitudes $\prod_{v} A_{v}\left(j_{\ell}, i_{v}\right)$, that reads [44, 46, 47, 51, 63, 69]:

$$
Z_{\mathcal{C}}=\sum_{j_{f}, i_{e}} \prod_{f} d\left(j_{f}\right) \prod_{v} A_{v}\left(j_{f}, \mathrm{v}_{e}\right)
$$

The vertex amplitude $A_{v}\left(j_{f}, \mathrm{v}_{e}\right)=\left\langle j_{f}, \mathrm{v}_{e} \mid A_{v}\right\rangle$ is written in a basis of intertwiners that diagonalizes the volume, and we indicate with $\mathrm{v}_{e}$ the corresponding quantum number, which we take to be the eigenvalue (for simplicity of notation we disregard the eventual degeneracy). Thus the vertex amplitude is a function of the spins $j_{f}$ and of the intertwiners adjacent to the vertex $v$.

We can include a positive cosmological constant by considering a simple modification of (4.1) based on the form of the cosmological-constant term in the Hamiltonian constraint. In the canonical theory, the cosmological constant appears as an additive term to the gravitational 
Hamiltonian constraint, which multiples the 3-volume element. When deriving a path integral formulation of quantum theory à la Feynman by inserting resolutions of unity into the evolution operator, a potential term appears simply as a multiplicative exponential, because the potential is diagonal in the position basis. The cosmological constant term is diagonal in the spin-intertwiner basis. It is therefore possible to insert the cosmological constant "potential" as a multiplicative term along the spinfoam evolution, that is in between 4-cells, which is to say on 3 -cells ${ }^{15}$. The coupling is therefore very simple, and consists in weighting edge amplitudes with an exponential term which depends on the volume and the cosmological constant. Therefore we obtain

$$
Z_{\mathcal{C}}=\sum_{j_{f}, \mathrm{v}_{e}} \prod_{f}(2 j+1) \prod_{e} e^{i \lambda \mathrm{v}_{e}} \prod_{v} A_{v}\left(j_{f}, \mathrm{v}_{e}\right),
$$

where $\lambda$ is related to the cosmological constant $\Lambda$ and eigenvalue of the volume $\mathrm{v}_{e}$ associated to an edge $e$. The amplitude is written in a basis of intertwiners that diagonalizes the volume, so that the term with $\mathrm{v}_{e}$ in the exponential is well defined.

Incorporating this term into the covariant dynamics of Loop Quantum Gravity (see [81, 87] and references therein) is important in order to check the semiclassical limit. Einstein equation admits only the trivial flat solution in absence of matter for $\Lambda=0$. Recovering flat space is interesting, but is still weak evidence for the full classical limit. Since at the moment the coupling of matter in spinfoam is not yet completely understood, the inclusion of the cosmological constant became essential to check the good semiclassical limit of the spinfoam theory beyond the trivial flat solution.

\subsubsection{Coherent states}

We want to study the semiclassical behavior of this transition amplitude. In in the Hilbert space $\mathcal{H}_{\Gamma}$ it is possible to define an overcomplete basis of semiclassical states, obtained as a superposition of spinnetwork states. These are coherent states, functions of SU(2) and labelled by a $\operatorname{SL}(2, \mathbb{C})$ element $H_{\ell}$ for each link. They take the form ${ }^{16}[29,30]$

$$
\psi_{H_{\ell}}\left(h_{\ell}\right)=\int_{\mathrm{SU}(2)^{N}} d g_{n} \prod_{l \in \Gamma} K_{t}\left(g_{s(\ell)} h_{\ell} g_{t(\ell)}^{-1} H_{\ell}^{-1}\right) .
$$

They are defined by an integral on $\mathrm{SU}(2)$, so that the stases are gauge invariant, and by the heat kernel $K_{t}$ on $\mathrm{SU}(2)$ ( $\left.h_{\ell} \in \mathrm{SU}(2)\right)$, analytically continued to $\mathrm{SL}(2, \mathbb{C})$. This is a function concentrated on the origin of the group, with a spread of order $1 / t$ in $j$. Its explicit form is ${ }^{17}$

$$
K_{t}(h)=\sum_{j}(2 j+1) e^{-2 t \hbar j(j+1)} \operatorname{Tr}\left[D^{j}(h)\right]
$$

where $D^{j}(h)$ is the Wigner matrix of the spin- $j$ representation of $\mathrm{SU}(2)$. The states (4.3) are gauge-invariant semiclassical wave packets. The integral in (4.3) projects ("group averages") on the gauge invariant states. If $H_{\ell}$ is in the $\mathrm{SU}(2)$ subgroup of $\mathrm{SL}(2, \mathbb{C})$, the heat kernel peaks each $h_{\ell}$ on $H_{\ell}$. The extension of $H_{\ell}$ to $\mathrm{SL}(2, \mathbb{C})$ has the same effect as taking a gaussian function

\footnotetext{
${ }^{15}$ The boundary state of each cell is written in the time gauge [95].

${ }^{16}$ As shown in [29], these states: (i) are the basis of the holomorphic representation [7, 30], (ii) are a special case of Thiemann's complexifier's coherent states [20, 21, 49, 50, 97, 98, 99, 104, 105, 106], (iii) induce Speziale-Livine coherent tetrahedra [36, 52, 69] on the nodes, and (iv) are equal to the Freidel-Speziale coherent states [55, 56] for large spins.

${ }^{17}$ We choose a parameter $t$ with the dimension of an inverse action, and put $\hbar$ explicitly in the definition of the coherent states, in order to emphasize the fact that the small $t$ limit is the classical limit, and to keep track of the corresponding dependence on $\hbar$. The factor 2 is for later convenience.
} 


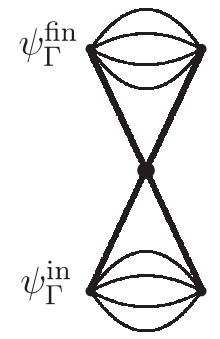

Figure 8. Transition amplitude between two states defined on a "dipole" graph. We consider only the first order in the vertex expansion, i.e. there is only one vertex in the bulk (spinfoam edges are drawn with thicker lines).

$\psi(x)=e^{\left(x-z_{o}\right)^{2} / 2} \sim e^{\left(x-x_{o}\right)^{2} / 2} e^{i p_{o} x}$ for a complex $z_{o}=x_{o}+i p_{o}$; that is, it adds a phase which peaks the states on a value of the variable conjugate to $h_{\ell}$. Thus, the states (4.3) are peaked on the variables $h_{\ell}$ as well as on their conjugate momenta.

We can decompose each $\mathrm{SL}(2, \mathbb{C})$ label in the form

$$
H_{\ell}=D^{(j)}\left(R_{\vec{n}_{s(\ell)}}\right) e^{-i z_{\ell} \frac{\sigma_{3}}{2}} D^{(j)}\left(R_{\vec{n}_{t(\ell)}^{-1}}^{-}\right),
$$

where $R_{\vec{n}} \in \mathrm{SU}(2)$ is the rotation matrix that rotates the unit vector pointing in the $(0,0,1)$ direction into the unit vector $\vec{n}$, and $D^{(j)}\left(R_{\vec{n}_{s}}\right)$ is its representation $j . \vec{\sigma}=\left\{\sigma_{i}\right\}, i=1,2,3$ are the Pauli matrices.

There is a compelling geometrical interpretation for the $\left(\vec{n}_{s}, \vec{n}_{t}, \xi, \eta\right)$ labels of each link [55, $72,94]$. The two vectors $\vec{n}_{s}$ and $\vec{n}_{t}$ represent the normals to the face $\ell$, in the two polyhedra bounded by this face. The complex number $z_{\ell}$ codes the intrinsic and the extrinsic geometry at the face. More precisely the imaginary part of $z_{\ell}$ is proportional to the area of the face of the cellular decomposition dual to the link $\ell$. The real part of $z_{\ell}$ is determined by the holonomy of the Ashtekar connection along the link [94]. For general states, the interpretation extends to a simple generalization of Regge geometries, that Freidel and Speziale have baptized "twisted geometries" [55].

These state, that we use to concretely compute the transition amplitude, should be interpreted $^{18}$ as describing the quantum space surrounding a given 4-dimensional finite region of spacetime. We talk therefore of "boundary states", that can be thought here as "in" and "out" states in the transition amplitude.

\subsubsection{Vertex amplitude}

The transition amplitude (4.1), (4.2) can be expressed as a vertex expansion. The first nontrivial term of this expansion involves just a single vertex. In what follows we concentrate on the evaluation of the transition amplitude in the first order of the vertex expansion. Therefore the vertex amplitudes $A_{v}\left(H_{\ell}\right)$, one for each vertex $v$ in the bulk of the spinfoam, become for us just $A\left(H_{\ell}\right)$. We evaluate it in the basis of the coherent states $[22,25,30,45,77]$, so that the expression $\left\langle A \mid \psi_{H_{\ell}}\right\rangle=W\left(H_{\ell}\right)$ reads

$$
\begin{aligned}
W\left(H_{\ell}\right)= & \int_{\mathrm{SL}(2, \mathbb{C})} \prod_{n=1}^{N-1} \mathrm{~d} G_{n} \prod_{\ell=1}^{L} \sum_{j_{\ell}}\left(2 j_{\ell}+1\right) e^{-2 t \hbar j_{\ell}\left(j_{\ell}+1\right)} e^{i \lambda_{\mathrm{v}_{e}}} \\
& \times \operatorname{Tr}\left[D^{\left(j_{\ell}\right)}\left(H_{\ell}\right) Y^{\dagger} D^{\left(\gamma j_{\ell}, j_{\ell}\right)}\left(G_{\ell}\right) Y\right] .
\end{aligned}
$$

\footnotetext{
${ }^{18}$ They can also be viewed as describing quantum space at some given coordinate time, but this interpretation is less covariant.
} 
The amplitude is Lorentzian, with an integration over the $\operatorname{SL}(2, \mathbb{C})$ elements $G_{n}$ associated to the edges (see Fig. 8). Notice that the integration is over all the $G_{n}$ but one, in order to avoid a redundancy that makes the amplitude diverge [45]. We take the product over each link $\ell$ and the sum over the coloring of these links, i.e. on the spins $j_{\ell} . D^{(j)}\left(H_{\ell}\right)$ is simply $D^{(j)}\left(R_{\vec{n}_{s(\ell)}}\right) D^{(j)}\left(e^{-i z_{\ell} \frac{\sigma_{3}}{2}}\right) D^{(j)}\left(R_{\vec{n}_{t(\ell)}}^{-1}\right) . G_{\ell}=G_{s(\ell)} G_{t(\ell)}^{-1}$ is the product of the $\mathrm{SL}(2, \mathbb{C})$ group elements at the source and target nodes, extremals of each oriented link $\ell$, and $D^{\left(\gamma j_{\ell}, j_{\ell}\right)}\left(G_{\ell}\right)$ is its representation matrix. Finally, $Y$ is a map from the representation $(j)$ of $\mathrm{SU}(2)$ to the representation $\left(\gamma j_{\ell}, j_{\ell}\right)$ of $\operatorname{SL}(2, \mathbb{C})$. The first has has dimension $2 j+1$ while the second has infinite dimension. These matrices with different dimensions are glued by the map $Y$. In other words

$$
\begin{aligned}
Y: \mathcal{H}^{(j)} & \longrightarrow \mathcal{H}^{(j, \gamma j)} \\
|j, m\rangle & |(j, \gamma j) ; j, m\rangle
\end{aligned}
$$

whose matrix elements are given by $\left\langle(j, \gamma j) ; j^{\prime}, m^{\prime}|Y| j, m\right\rangle=\delta_{p, \gamma j} \delta_{k j} \delta_{j j^{\prime}} \delta_{m m^{\prime}}$.

In the base of the coherent states the amplitude takes the convenient form (4.4) that we exploit for the calculation in cosmology.

\subsection{Homogeneous and isotropic geometry}

We want to evaluate the vertex amplitude (4.4) for the homogeneous and isotropic case [28, 31, 107, 108]. This corresponds to restrict the study to regular graphs, i.e. graphs where the distribution of the degrees at the nodes is uniform (this condition is trivially satisfy when we work with the dipole). The requirement of homogeneity and isotropy fixes $\vec{n}_{s}, \vec{n}_{n}$ as the normals to the faces of the geometrically regular cellular decomposition dual to the graph, and implies that all the $z_{\ell}$ elements in $H_{\ell}$ are equal: $z_{\ell}=z$. Furthermore, on a homogeneous isotropic space the real part of $z$ is the sum of two terms [71] $\operatorname{Re} z=\theta(\gamma K+\Gamma)$, where $K$ and $\Gamma$ are the scalar coefficients of respectively the extrinsic curvature and the spin connection, that enter in the definition of the Ashtekar-Barbero connection written in the homogeneous gauge. On a compact space, $\Gamma=1$, and $\theta$ and is the angle between two $4 \mathrm{~d}$ normals of the two adjacent polyhedra (the isotropy requires that this is the same for every coupe of normals) and $K$ is proportional to the time derivative of the scale factor. Finally, all the cells are equal and we can write $\mathrm{v}_{e}$ in the cosmological constant term as the volume $\mathrm{v}_{o}$ of a regular cell with faces having unit area, times $j^{\frac{3}{2}}$.

With these assumptions, any homogeneous isotropic coherent state on any regular graph is described by a single complex variable $z$, whose imaginary part is proportional to the area of each regular face of the cellular decomposition (and it can be put in correspondence with the total volume) and whose real part is related to the extrinsic curvature [94]. We denote $\psi_{H_{\ell}(z)}$ this state, and $\psi_{H_{\ell}\left(z, z^{\prime}\right)}=\psi_{H_{\ell}(z)} \otimes \psi_{H_{\ell}\left(z^{\prime}\right)}$ the state on two copies of the regular graph, obtained tensoring the "in" and "out" homogeneous isotropic states.

Before studying further our transition amplitude, let us consider the vertex expansion. We consider the classical Hamilton function of a homogeneous isotropic cosmology: this results in a difference between two boundary terms. With the cosmological constant $\Lambda$ it gives

$$
S_{H}=\left.\int \mathrm{d} t\left(a \dot{a}^{2}+\frac{\Lambda}{3} a^{3}\right)\right|_{\dot{a}= \pm \sqrt{\frac{\Lambda}{3}} a}=\frac{2}{3} \sqrt{\frac{\Lambda}{3}}\left(a_{\mathrm{fin}}^{3}-a_{\mathrm{in}}^{3}\right),
$$

where $a$ is the scale factor and $\dot{a}$ its time derivative. Therefore at the first order in $\hbar$ the quantum transition amplitude factorizes:

$$
W\left(a_{\mathrm{fin}}, a_{\mathrm{in}}\right)=e^{\frac{i}{\hbar} S_{H}\left(a_{\mathrm{fin}}, a_{\mathrm{in}}\right)}=W\left(a_{\mathrm{fin}}\right) \overline{W\left(a_{\mathrm{in}}\right)} .
$$


The same happens for the spinfoam amplitude

$$
\left\langle W \mid \psi_{H_{\ell}\left(z_{\text {fin }}, z_{\text {in }}\right)}\right\rangle=W\left(z_{\text {fin }}, z_{\text {in }}\right)=W\left(z_{\text {fin }}\right) \overline{W\left(z_{\text {in }}\right)}
$$

with $W(z) \equiv W\left(H_{\ell}\right)$, where now (4.4) edpends only on a single $z$ trough $H_{\ell}(z)$.

In [60], Frank Hellmann points out that the factorization survives also beyond the classical (large distance) limit when we restrict to the one-vertex approximation of the amplitude, and observes that this factorization can be reinterpreted as the amplitude to go from the initial state to nothing and from nothing to the final state, namely as the contribution of a disconnected spacetime topology to the total transition amplitude. This does not preclude assigning a standard cosmological interpretation to the $W\left(z_{\text {fin }}, z_{\text {in }}\right)=W\left(z_{\text {fin }}\right) \overline{W\left(z_{\text {in }}\right)}$ amplitude: it is only a consequence of the particularly simple dynamics of the classical symmetric system considered above, where the equations of motion determine a relation between $a$ and its conjugate momentum $p_{a}$ which is independent from earlier values of the two. On the other hand, this observation is quite interesting because it allows each single term $W(z)$ to be interpreted as the HartleHawking "wave function of the universe" determined by a no-boundary initial condition [59]. At the first order in the expansion, we can therefore study $W(z)$ instead of $W\left(z_{\mathrm{fin}}, z_{\text {in }}\right)$ and interpret it as the "wave function of the universe".

We are interested in this quantity in the large volume limit, that correspond to take the imaginary part of $z$ is large. Let us consider separately the real and the imaginary part of $z$.

When the imaginary part of $z$ is large we find that the Wigner matrix in the trace gives

$$
D^{(j)}\left(e^{-i z \frac{\sigma_{3}}{2}}\right)=\sum_{m} e^{-i z m}|m\rangle\langle m| .
$$

For $\operatorname{Im} z \gg 1$ (large area) in this sum the term $m=j$ dominates, therefore

$$
D^{(j)}\left(e^{-i z \frac{\sigma_{3}}{2}}\right) \approx e^{i z j}|j\rangle\langle j|
$$

where $|j\rangle$ is the eigenstate of $L_{3}$ with maximum eigenvalue $m=j$ in the representation $j$. Inserting this result into (4.4) we obtain

$$
\begin{aligned}
W(z)= & \int \prod_{n=1}^{N-1} \mathrm{~d} G_{n} \prod_{\ell=1}^{L} \sum_{j_{\ell}}\left(2 j_{\ell}+1\right) e^{-2 t \hbar j_{\ell}\left(j_{\ell}+1\right)-i \lambda \mathrm{v}_{o} j^{\frac{3}{2}}-i z_{\ell} j_{\ell}} \\
& \times\left\langle j_{\ell}\left|D^{\left(j_{\ell}\right)}\left(R_{\vec{n}_{n}}^{-1}\right) Y^{\dagger} D^{\left(\gamma j_{\ell}, j_{\ell}\right)}\left(G_{\ell}\right) Y D^{\left(j_{\ell}\right)}\left(R_{\vec{n}_{s}}\right)\right| j_{\ell}\right\rangle .
\end{aligned}
$$

The action of the matrix $D^{\left(j_{\ell}\right)}\left(R_{\vec{n}_{n}}\right)$ on the highest weights states is precisely the definition of the coherent states $|\vec{n}\rangle$, so we can write

$$
\begin{aligned}
W(z)= & \int \prod_{n=1}^{N-1} \mathrm{~d} G_{n} \prod_{\ell=1}^{L} \sum_{j_{\ell}}\left(2 j_{\ell}+1\right) e^{-2 t \hbar j_{\ell}\left(j_{\ell}+1\right)-i \lambda \mathrm{v}_{o} j^{\frac{3}{2}}-i z_{\ell} j_{\ell}} \\
& \times\left\langle\vec{n}_{t(\ell)}\left|Y^{\dagger} D^{\left(\gamma j_{\ell}, j_{\ell}\right)}\left(G_{\ell}\right) Y\right| \vec{n}_{s(\ell)}\right\rangle .
\end{aligned}
$$

We can now study the $\operatorname{SL}(2, \mathbb{C})$ integral in (4.5) (without fixing the $j$ ). Let us rewrite the previous expression as

$$
\begin{aligned}
W(z)= & \sum_{\left\{j_{\ell}\right\}} \prod_{\ell=1}^{L}\left(2 j_{\ell}+1\right) e^{-2 t \hbar j_{\ell}\left(j_{\ell}+1\right)-i \lambda \mathrm{v}_{o} j^{\frac{3}{2}}-i z j_{\ell}} \\
& \times \int \prod_{n=1}^{N-1} \mathrm{~d} G_{n} \prod_{\ell=1}^{L}\left\langle\vec{n}_{t(\ell)}\left|Y^{\dagger} D^{\left(\gamma j_{\ell}, j_{\ell}\right)}\left(G_{\ell}\right) Y\right| \vec{n}_{s(\ell)}\right\rangle .
\end{aligned}
$$


Since the gaussian sums in the first line peak the $j_{\ell}$ 's over large values, the integral in the second line can be computed in the large spin regime, where it can be evaluated using saddle point methods. The computation of the integral in (4.6) can be written in a spinor base, as the one introduced in [25] and gives

$$
\int \prod_{n=1}^{N-1} \mathrm{~d} G_{n} \prod_{\ell=1}^{L}\left\langle n_{s(\ell)}\left|Y^{\dagger} D^{\left(\gamma j_{\ell}, j_{\ell}\right)}\left(G_{\ell}\right) Y\right| n_{t(\ell)}\right\rangle=\mathrm{H} \prod_{\ell=1}^{L} e^{-\frac{1}{2} i j_{\ell} \theta},
$$

where $\mathrm{H}$ is the Hessian of the logarithm of the integrand in (4.7) [25] and $\theta$ is a constant determined by the normals on the faces: it is the intrinsic curvature on the faces, coming from the spin connection in the Ashtekar connection. We can define a new variable $\tilde{z}:=z-\theta$, so that the real part of $\tilde{z}$ is exactly the extrinsic curvature.

We can now compute the sum that appears in the amplitude

$$
W(z)=\sum_{\{j\}} \mathrm{H} \prod_{\ell=1}^{L}\left(2 j_{\ell}+1\right) e^{-2 t \hbar j_{\ell}\left(j_{\ell}+1\right)-i \lambda \mathrm{v}_{o} j^{\frac{3}{2}}-i \tilde{z} j_{\ell}}
$$

by approximating it with a Gaussian integral peaked on $j_{\ell} \sim j_{o}$. We expand around $j_{0}$ so that the new term is $i \lambda \mathrm{v}_{o} j^{\frac{3}{2}} \sim i \lambda \mathrm{v}_{o} j_{o}^{\frac{3}{2}}+\frac{3}{2} i \lambda \mathrm{v}_{o} j_{o}^{\frac{1}{2}} \delta j$. The first term is a constant that can be reabsorbed in the normalization and the second contributes to the phase. The value of the peak of the gaussian $j_{o}$ is determined by the stationary point where the real part of the exponent in (4.8) vanishes. This gives a condition on the imaginary part of $\tilde{z}$ (associated to the area), that for large $(j \gg 1)$ is

$$
j_{o} \sim \operatorname{Im} \tilde{z} / 4 t \hbar .
$$

The imaginary part of (4.8) is a phase that suppress the amplitude everywhere but where the argument is zero or a multiple of $2 \pi$. This gives the condition

$$
\operatorname{Re} \tilde{z}=-\frac{3}{2} \lambda \mathrm{v}_{o} j^{\frac{1}{2}}
$$

that, together with the condition (4.9), becomes

$$
\operatorname{Re} \tilde{z}=-\frac{3}{2} \lambda \mathrm{v}_{o} j_{o}^{\frac{1}{2}}=-\frac{3}{2} \lambda \mathrm{v}_{o} \sqrt{\operatorname{Im} \tilde{z} / 4 t \hbar} .
$$

This expression yields the Friedmann equation: recall that $\operatorname{Re} \tilde{z} \sim \dot{a}$ and $\operatorname{Im} \tilde{z} \sim a^{2}$ so that, squaring the previous equation, we obtain

$$
\left(\frac{\dot{a}}{a}\right)^{2}=\frac{\Lambda}{3}
$$

where $\Lambda=27 \lambda^{2} \mathrm{v}_{o}^{2} / 16 t \hbar$. The same result can be obtained by a different technique: the transition amplitude results to be annichilated by a Hamiltonian constraint. In the classical limit, this is

$$
\left(\tilde{z}+\frac{3}{2} \lambda \mathrm{v}_{o} j_{o}^{\frac{1}{2}}\right)^{2}+\overline{\left(\tilde{z}+\frac{3}{2} \lambda \mathrm{v}_{o} j_{o}^{\frac{1}{2}}\right)^{2}}=0
$$

that gives

$$
i 4 \operatorname{Im} \tilde{z}\left(\operatorname{Re} \tilde{z}+\frac{3}{2} \lambda \mathrm{v}_{o} j_{o}^{\frac{1}{2}}\right)=0 .
$$

Notice that we don't obtain the curvature term $k / a^{2}$ in the full Friedmann equation

$$
\left(\frac{\dot{a}}{a}\right)^{2}=\frac{\Lambda}{3}-\frac{k}{a^{2}}
$$


This is because of the approximation taken in the evaluation of the gaussian sum. Since we ask for large $j$, namely for a large distance regime, the curvature term is neglected being a higher order in $j$. Finding a way to relax this approximation is an urgent issue in spinfoam cosmology: the higher order in $j$ would in fact provide us also the first quantum corrections. Christian Röken has observed that equation (4.10), including the curvature term, can be obtained simply by keeping the +1 term of the $\left(j_{\ell}+1\right)$ in $(4.8)$ and then rescaling $a$ appropriately [80].

\subsection{Graph independence and 2-node model}

As mentioned above, spinfoam cosmology was introduced in [31] with a calculation based on the dipole graph with 4 links. This choice was dictated by simplicity and by the fact that this graph, already studied in the canonical context, has a compelling interpretation being a triangulation of the 3-sphere. But the computation above shows that, studying the large distance limit the results for the dipole are the same as for other regular graphs. (We emphasize the fact that numerical investigation shows that the large $j$ convergence is very fast, and the asymptotic regime is already essentially reached with $j \sim 3$.) Let us discuss the terms in (4.8) that carry a dependence on the graph used.

At the stationary point the Hessian $\mathrm{H}$ give a contribution $N_{\Gamma}$ that depends on the graph $\Gamma$ trough its numbers of links $L$ and nodes $N$, and a characteristic term $j_{o}^{-3}$ that is independent of the graph. This is the norm squared of the Livine-Speziale coherent regular cell of size $j_{0}$ [69] (recently calculated for the Lorentzian signature [25]). Notice that since we have fixed the normals, degenerate contributions are not allowed (being these present, we would have had further terms $\sim j_{o}^{-1}$ ).

The volume $\mathrm{v}_{o}$ depends on the graph used. On the other hand, such a cosmological-constant term has been introduced as an edge amplitude. This edge amplitude can be viewed as a redefinition of the vertex. Possible normalization ambiguities, coming from the introduction of this term, can therefore be absorbed in the vertex amplitude [72].

The transition amplitudes that we are dicussing are in fact not normalized. The arbitrary normalization of the vertex amplitude is fixed by cylindrical consistency [72]. We find that the dependence on the number of nodes enters only in the term $N_{\Gamma}$ in (4.11), and it can be counterbalanced by normalizing appropriately the amplitude. This implies that this result can be obtained also in the 2-node model, with the only caveat that $N_{\Gamma}$ would be the one for 2 nodes.

Let us consider now the number of links. In the semiclassical limit the expression of the amplitude can be given in the form

$$
W(\tilde{z})=\left(2 j_{o} \sqrt{\frac{\pi}{t}} e^{-\frac{\tilde{z}^{2}}{8 t \hbar}}\right)^{L} \frac{N_{\Gamma}}{j_{o}^{3}} .
$$

Here the information about the semiclassical dynamics is coded in the kernel of the exponential, that does not depends on the number of links.

Using this and (4.9), we conclude

$$
W(\tilde{z})=N \tilde{z}^{L-3} e^{-\frac{L}{2 t \hbar} \tilde{z}^{2}}
$$

where $N=\left(\frac{4 \pi}{t}\right)^{L / 2}\left(\frac{-i}{4 t \hbar}\right)^{L-3} N_{\Gamma}$. Finally, inserting into (4.2) we have

$$
W\left(\tilde{z}_{\text {in }}, \tilde{z}_{\text {fin }}\right)=N^{2}\left(\tilde{z}_{\text {in }} \tilde{z}_{\text {fin }}\right)^{L-3} e^{-\frac{L}{2 t \hbar}\left(\tilde{z}_{\text {in }}^{2}+\tilde{z}_{\text {fin }}^{2}\right)} .
$$

This is the transition amplitude between two cosmological homogeneous isotropic coherent states, with $N$ and $L$ links such that the graph is regular (i.e. every node has the same valency). 
Notice that there are an infinite number of such graphs. For instance, two nodes can be connected by arbitrary number of links. Examples of regular graphs with $N>2$ are given by the (dual of) the Platonic solids.

The result of this calculation is that the support of the transition amplitude, obtained trough the conditions on the real and the imaginary part of $\tilde{z}$ that yields the Friedmann equation, is not sensitive to the number of links or the number of nodes of the graph used.

One aspect of the problem that (to the best of our knowledge) has not yet been studied is to compare the transition amplitude for homogeneous to homogeneous geometries to the transition amplitude from homogeneous to inhomogeneous geometries on the same graph. Namely to understand wether the quantum evolution smears out a state that is peaked on a homogeneous geometry to one roughly equally distributed over all geometries.

\subsubsection{Covariant $\mathrm{U}(N)$ framework}

In Section 3, we have illustrated the power of the $\mathrm{U}(N)$ framework, for instance for the implementation of homogeneity and isotropy in the 2-node model. Is it possible to use this technique in the covariant theory illustrated in this section? Work in this directions is still under development. A first step has been recently proposed in [68], where $\mathrm{U}(N)$ coherent states are defined on the dipole (Fig. 5) and a simple $\mathrm{SU}(2)$ transition amplitude between these states is studied. This is an interesting research direction that deserves further studies. In particular, the next step should be to include in the picture the full Lorentzian spinfoam dynamics of general relativity.

\section{Summary}

In the early days of LQG, the realization that the theory lead to Planck scale discreteness nourished the intuition that the only way of recovering a continuous space from the theory was to have a very large number of links $[11,62]$. Gaining clarity about the distinction between large number of links and large quantum numbers such as the area quantum number $j$, lead to the realization that the theory can describe large semiclassical geometries also over a small number of nodes and links. Here we have reviewed a number of constructions in Loop Quantum Gravity, based on the idea of truncating the Hilbert space of the theory down to the states supported on a simple graph with two nodes [96].

The restriction of the full LQG Hilbert space to a simple graph is a truncation of the degrees of freedom of the full theory. It defines an approximation where concrete calculations can be performed. The approximation is viable in physical situations where only a small number of the degrees of freedom of General Relativity are relevant. A characteristic example is cosmology.

The 2-node graphs (dipole) with 4 links defines the simplest triangulation of a 3-sphere and can accommodate the anisotropic degrees of freedom of a Bianchi IX model, plus some inhomogeneous degrees of freedom, which can be seen as the lowest modes in a spherical-harmonic expansion, following a technique introduced by Regge and Hu. In this context, a Bohr-Oppenheimer approximation provides a tool to separate heavy and light degrees of freedom, and extract the FLRW dynamics. This way of deriving quantum cosmology from LQG is different from the usual one: in standard loop quantum cosmology, the strategy is to start from a symmetry-reduced system, and quantize the single or the few degrees of freedom that survive in the symmetry reduction. Here instead we consider a truncated version of the full quantum theory of gravity, in the LQG framework, and look for a "cosmological sector" inside the theory.

In Section 3 we have stepped up to a 2-node graphs with arbitrary number of links. This system provides an immediate application of the $\mathrm{U}(N)$ formalism [32]. This formalism is based on the observation that the LQG Hilbert space of intertwiners with $N$-legs and fixed area is an irreducible representation of the group $\mathrm{U}(N)$ [53]. The relation is made explicit using the 
Schwinger representation of SU(2). Furthermore, the full state space of $N$-leg intertwiners can be endowed with a Fock-space structure, with annihilation and creation operators $F_{i j}$ and $F_{i j}^{\dagger}$.

In the 2-node graph context, one can define operators $e_{i j}$ and $f_{i j}$, that are $\mathrm{SU}(2)$ invariant and consistent with the matching conditions between the intertwiners (ensuring that the spin number of one leg is the same seen from both nodes). The system has a $\mathrm{U}(N)$ global symmetry, given by a generalization of the matching conditions. The space of the states invariant under this symmetry is homogeneous and isotropic. This construction defines the homogeneous and isotropic configurations via a symmetry reduction at the quantum level, and may shed further light on the relation between Loop Quantum Cosmology and Loop Quantum Gravity. In particular, the construction leads to the definitions of a nontrivial consistent Hamiltonian operator for the homogeneous/isotropic sector, which has intriguing mathematical analogies with the operators used in LQC.

We have also reviewed the classical spinor system whose quantization yields the Hilbert space of intertwiners of LQG [34, 70]. This framework permits the construct of the classical counterpart of the $\mathrm{U}(N)$ Hamiltonian for the 2-node model, defining an effective classical dynamics for this system. The equations of motion of this classical system can be solved, and the resulting dynamics shows analogies with the results of LQC.

In Section 4 we switched to the covariant, or spinfoam, definition of the dynamics. Here the dynamics isnot defined by a Hamiltonian, but rather directly by a transition amplitude between two states of the quantum geometry. At the first order in the vertex expansion, this amplitude factorizes and defines a "wave function of the universe" à la Hartle-Hawking. In the classical limit, the amplitude turns out to be peaked on the solutions of Einstein equations, that is, In presence of isotropy and homogeneity, of the Friedmann equation [31]. The model can include the presence of the cosmological constant [28]. This is obtained by inserting a "face amplitude" term into the spinfoam amplitude and can be seen as an effective way to include $\Lambda$. It is generally thought that at the fundamental level the cosmological constant should emerge in a quantum deformed version of the spinfoam theory. Such a quantum deformation should hopefully results in a term that match with the one that we have heuristically introduced.

The amplitude for homogeneous isotropic states was first computed using the dipole graph, but, remarkably, the classical limit of the amplitude turns out to be independ on the (regular) graph chosen [107]. This result supports the viability of the approximation taken by restricting the theory to a single graph. States of large regular graphs include in principle inhomogeneous quantum fluctuations, beyond perturbations techniques as usually utilized in quantum cosmology.

In closing, we point out three directions where the techniques reviewed here might turn out to be useful to better understand loop cosmology.

First, the $\mathrm{U}(N)$ symmetry provides an elegant way to impose inhomogeneity and anisotropy and so far the attention has focused on the $\mathrm{U}(N)$-invariant states. Can we go beyond this sector? Indeed, the action that defines the full classical kinematics and dynamics of spinnetwork states on the 2-node graph is a nontrivial matrix model defined in terms of the unitary matrices $U^{\alpha}$ and $U^{\beta}$, with quartic interaction terms. It would be very interesting to see what kind of anisotropy does our model describe in the context of loop cosmology.

Second, the relation between the different dynamics defined by standard LQC, by the Hamiltonian of the $\mathrm{U}(N)$ framework and by the spinfoam amplitude need to be compared in detail. For this, in particular, he analysis of the spinfoam amplitude should be developed beyond the semiclassical limit.

Finally, all the analysis reviewed here is in the context of pure gravity, and disregard the presence of matter. The coupling of fermions and Yang-Mills fields is simple and natural in the Hilbert space of Loop Quantum Gravity at the kinematical level. In the $\mathrm{U}(N)$ approach there is a direct formulation of this coupling in spinor phase space before quantization. 
The coupling of fermions to canonical LQG is well understood $[15,74,75,100,101]$. In the spinfoam approach, the dynamical coupling with fermions and Yang-Mills fields has been defined recently in $[27,58]$ and has not yet been much studied. Including matter couplings is clearly essential for understanding the quantum dynamics of cosmology.

\section{Acknowledgements}

This work was in part supported by the Spanish MICINN research grants FIS2008-01980 and FIS2009-11893. IG is supported by the Department of Education of the Basque Government under the "Formación de Investigadores" program.

\section{References}

[1] Ashtekar A., An introduction to loop quantum gravity through cosmology, Nuovo Cim. B 122 (2007), 135-155, gr-qc/0702030.

[2] Ashtekar A., Lectures on nonperturbative canonical gravity, Advanced Series in Astrophysics and Cosmology, Vol. 6, World Scientific Publishing Co. Inc., River Edge, NJ, 1991.

[3] Ashtekar A., New variables for classical and quantum gravity, Phys. Rev. Lett. 57 (1986), 2244-2247.

[4] Ashtekar A., Bojowald M., Lewandowski J., Mathematical structure of loop quantum cosmology, Adv. Theor. Math. Phys. 7 (2003), 233-268, gr-qc/0304074.

[5] Ashtekar A., Corichi A., Singh P., Robustness of key features of loop quantum cosmology, Phys. Rev. D $7 \mathbf{7}$ (2008), 024046, 17 pages, arXiv:0710.3565.

[6] Ashtekar A., Lewandowski J., Representation theory of analytic holonomy $C^{*}$-algebras, in Knots and Quantum Gravity (Riverside, CA, 1993), Oxford Lecture Ser. Math. Appl., Vol. 1, Oxford Univ. Press, New York, 1994, 21-61, gr-qc/9311010.

[7] Ashtekar A., Lewandowski J., Marolf D., Mourão J., Thiemann T., Coherent state transforms for spaces of connections, J. Funct. Anal. 135 (1996), 519-551, gr-qc/9412014.

[8] Ashtekar A., Pawlowski T., Singh P., Quantum nature of the big bang, Phys. Rev. Lett. 96 (2006), 141301, 4 pages, gr-qc/0602086.

[9] Ashtekar A., Pawlowski T., Singh P., Quantum nature of the big bang: improved dynamics, Phys. Rev. D 74 (2006), 084003, 23 pages, gr-qc/0607039.

[10] Ashtekar A., Pawlowski T., Singh P., Vandersloot K., Loop quantum cosmology of $k=1$ FRW models, Phys. Rev. D 75 (2007), 024035, 26 pages, gr-qc/0612104.

[11] Ashtekar A., Rovelli C., Smolin L., Weaving a classical metric with quantum threads, Phys. Rev. Lett. 69 (1992), 237-240, hep-th/9203079.

[12] Ashtekar A., Singh P., Loop quantum cosmology: a status report, Classical Quantum Gravity 28 (2011), 213001, 122 pages, arXiv:1108.0893.

[13] Baez J.C., Spin networks in gauge theory, Adv. Math. 117 (1996), 253-272, gr-qc/9411007.

[14] Baez J.C., Spin networks in nonperturbative quantum gravity, in The Interface of Knots and Physics (San Francisco, CA, 1995), Proc. Sympos. Appl. Math., Vol. 51, Editor L.H. Kauffman, Amer. Math. Soc., Providence, RI, 1996, 167-203, gr-qc/9504036.

[15] Baez J.C., Krasnov K.V., Quantization of diffeomorphism-invariant theories with fermions, J. Math. Phys. 39 (1998), 1251-1271, hep-th/9703112.

[16] Bahr B., Dittrich B., (Broken) gauge symmetries and constraints in Regge calculus, Classical Quantum Gravity 26 (2009), 225011, 34 pages, arXiv:0905.1670.

[17] Bahr B., Dittrich B., Improved and perfect actions in discrete gravity, Phys. Rev. D 80 (2009), 124030, 15 pages, arXiv:0907.4323.

[18] Bahr B., Dittrich B., He S., Coarse graining free theories with gauge symmetries: the linearized case, New J. Phys. 13 (2011), 045009, 34 pages, arXiv:1011.3667.

[19] Bahr B., Dittrich B., Steinhaus S., Perfect discretization of reparametrization invariant path integrals, Phys. Rev. D 83 (2011), 105026, 19 pages, arXiv:1101.4775. 
[20] Bahr B., Thiemann T., Gauge-invariant coherent states for loop quantum gravity. I. Abelian gauge groups, Classical Quantum Gravity 26 (2009), 045011, 22 pages, arXiv:0709.4619.

[21] Bahr B., Thiemann T., Gauge-invariant coherent states for loop quantum gravity. II. Non-Abelian gauge groups, Classical Quantum Gravity 26 (2009), 045012, 45 pages, arXiv:0709.4636.

[22] Barrett J.W., Dowdall R.J., Fairbairn W.J., Hellmann F., Pereira R., Lorentzian spin foam amplitudes: graphical calculus and asymptotics, Classical Quantum Gravity 27 (2010), 165009, 34 pages, arXiv:0907.2440.

[23] Battisti M.V., Marcianó A., Big bounce in dipole cosmology, Phys. Rev. D 82 (2010), 124060, 5 pages, arXiv:1010.1258.

[24] Battisti M.V., Marcianò A., Rovelli C., Triangulated loop quantum cosmology: Bianchi IX universe and inhomogeneous perturbations, Phys. Rev. D 81 (2010), 064019, 16 pages, arXiv:0911.2653.

[25] Bianchi E., Ding Y., Lorentzian spinfoam propagator, arXiv:1109.6538.

[26] Bianchi E., Dona' P., Speziale S., Polyhedra in loop quantum gravity, Phys. Rev. D 83 (2011), 044035 , 17 pages, arXiv:1009.3402.

[27] Bianchi E., Han M., Magliaro E., Perini C., Rovelli C., Wieland W., Spinfoam fermions, arXiv:1012.4719.

[28] Bianchi E., Krajewski T., Rovelli C., Vidotto F., Cosmological constant in spinfoam cosmology, Phys. Rev. D 83 (2011), 104015, 4 pages, arXiv:1101.4049.

[29] Bianchi E., Magliaro E., Perini C., Coherent spin-networks, Phys. Rev. D 82 (2010), 024012, 7 pages, arXiv:0912.4054.

[30] Bianchi E., Magliaro E., Perini C., Spinfoams in the holomorphic representation, Phys. Rev. D 82 (2010), 124031, 10 pages, arXiv:1004.4550.

[31] Bianchi E., Rovelli C., Vidotto F., Towards spinfoam cosmology, Phys. Rev. D 82 (2010), 084035, 8 pages, arXiv:1003.3483.

[32] Borja E.F., Díaz-Polo J., Garay I., Livine E.R., Dynamics for a 2-vertex quantum gravity model, Classical Quantum Gravity 27 (2010), 235010, 34 pages, arXiv:1006.2451.

[33] Borja E.F., Díaz-Polo J., Garay I., Livine E.R., U(n) invariant dynamics for a simplified loop quantum gravity model, arXiv:1012.3832.

[34] Borja E.F., Freidel L., Garay I., Livine E.R., U(N) tools for loop quantum gravity: the return of the spinor, Classical Quantum Gravity 28 (2011), 055005, 28 pages, arXiv:1010.5451.

[35] Collins P.A., Williams R.M., Dynamics of the Friedmann universe using Regge calculus, Phys. Rev. D 7 (1973), 965-971.

[36] Conrady F., Freidel L., Quantum geometry from phase space reduction, J. Math. Phys. 50 (2009), 123510, 29 pages, arXiv:0902.0351.

[37] Corichi A., Singh P., Is loop quantization in cosmology unique?, Phys. Rev. D 78 (2008), 024034, 13 pages, arXiv:0805.0136.

[38] Dittrich B., Partial and complete observables for canonical general relativity, Classical Quantum Gravity 23 (2006), 6155-6184, gr-qc/0507106.

[39] Dittrich B., Partial and complete observables for Hamiltonian constrained systems, Gen. Relativity Gravitation 39 (2007), 1891-1927, gr-qc/0411013.

[40] Dittrich B., Tambornino J., Gauge-invariant perturbations around symmetry-reduced sectors of general relativity: applications to cosmology, Classical Quantum Gravity 24 (2007), 4543-4585, gr-qc/0702093.

[41] Domagala M., Giesel K., Kamiński W., Lewandowski J., Gravity quantized: loop quantum gravity with a scalar field, Phys. Rev. D 82 (2010), 104038, 13 pages, arXiv:1009.2445.

[42] Dupuis M., Livine E.R., Revisiting the simplicity constraints and coherent intertwiners, Classical Quantum Gravity 28 (2011), 085001, 36 pages, arXiv:1006.5666.

[43] Dürr S., Fodor Z., Frison J., Hoelbling C., Hoffmann R., Katz S.D., Krieg S., Kurth T., Lellouch L., Lippert T., Szabo K.K., Vulvert G., Ab initio determination of light hadron masses, Science 322 (2008), 1224-1227, arXiv:0906.3599.

[44] Engle J., Livine E., Pereira R., Rovelli C., LQG vertex with finite Immirzi parameter, Nuclear Phys. B 799 (2008), 136-149, arXiv:0711.0146.

[45] Engle J., Pereira R., Regularization and finiteness of the Lorentzian loop quantum gravity vertices, Phys. Rev. D 79 (2009), 084034, 10 pages, arXiv:0805.4696. 
[46] Engle J., Pereira R., Rovelli C., Flipped spinfoam vertex and loop gravity, Nuclear Phys. B 798 (2008), 251-290, arXiv:0708.1236.

[47] Engle J., Pereira R., Rovelli C., Loop-quantum-gravity vertex amplitude, Phys. Rev. Lett. 99 (2007), 161301, 4 pages, arXiv:0705.2388.

[48] Fleischhack C., Irreducibility of the Weyl algebra in loop quantum gravity, Phys. Rev. Lett. 97 (2006), 061302, 4 pages.

[49] Flori C., Semiclassical analysis of the loop quantum gravity volume operator: area coherent states, arXiv:0904.1303.

[50] Flori C., Thiemann T., Semiclassical analysis of the loop quantum gravity volume operator. I. Flux coherent states, arXiv:0812.1537.

[51] Freidel L., Krasnov K., A new spin foam model for 4D gravity, Classical Quantum Gravity 25 (2008), 125018, 36 pages, arXiv:0708.1595.

[52] Freidel L., Krasnov K., Livine E.R., Holomorphic factorization for a quantum tetrahedron, Comm. Math. Phys. 297 (2010), 45-93, arXiv:0905.3627.

[53] Freidel L., Livine E.R., The fine structure of SU(2) intertwiners from U(N) representations, J. Math. Phys. 51 (2010), 082502, 19 pages, arXiv:0911.3553.

[54] Freidel L., Livine E.R., U(N) coherent states for loop quantum gravity, J. Math. Phys. 52 (2011), 052502, 21 pages, arXiv:1005.2090.

[55] Freidel L., Speziale S., Twisted geometries: a geometric parametrization of SU(2) phase space, Phys. Rev. D 82 (2010), 084040, 16 pages, arXiv:1001.2748.

[56] Freidel L., Speziale S., Twistors to twisted geometries, Phys. Rev. D 82 (2010), 084041, 5 pages, arXiv:1006.0199.

[57] Girelli F., Livine E.R., Reconstructing quantum geometry from quantum information: spin networks as harmonic oscillators, Classical Quantum Gravity 22 (2005), 3295-3313, gr-qc/0501075.

[58] Han M., Rovelli C., Spinfoam fermions: PCT symmetry, Dirac determinant, and correlation functions, arXiv:1101.3264.

[59] Hartle J.B., Hawking S.W., Wave function of the universe, Phys. Rev. D 28 (1983), 2960-2975.

[60] Hellmann F., Expansions in spin foam cosmology, Phys. Rev. D 84 (2011), 103516, 9 pages, arXiv:1105.1334.

[61] Hu B.L., Regge T., Perturbations on the mixmaster universe, Phys. Rev. Lett. 29 (1972), 1616-1620.

[62] Iwasaki J., Rovelli C., Gravitons as embroidery on the weave, Internat. J. Modern Phys. D 1 (1992), 533-557.

[63] Kamiński W., Kisielowski M., Lewandowski J., Spin-foams for all loop quantum gravity, Classical Quantum Gravity 27 (2010), 095006, 24 pages, arXiv:0909.0939.

[64] Kamiński W., Lewandowski J., Pawłowski T., Physical time and other conceptual issues of quantum gravity on the example of loop quantum cosmology, Classical Quantum Gravity 26 (2009), 035012, 20 pages, arXiv:0809.2590.

[65] Kisielowski M., Lewandowski J., Puchta J., Feynman diagrammatic approach to spin foams, arXiv:1107.5185.

[66] Krajewski T., Magnen J., Rivasseau V., Tanasa A., Vitale P., Quantum corrections in the group field theory formulation of the Engle-Pereira-Rovelli-Livine and Freidel-Krasnov models, Phys. Rev. D 82 (2010), 124069, 20 pages, arXiv:1007.3150.

[67] Lewandowski J., Okołów A., Sahlmann H., Thiemann T., Uniqueness of diffeomorphism invariant states on holonomy-flux algebras, Comm. Math. Phys. 267 (2006), 703-733, gr-qc/0504147.

[68] Livine E.R., Martín-Benito M., Classical setting and effective dynamics for spinfoam cosmology, arXiv:1111.2867.

[69] Livine E.R., Speziale S., New spinfoam vertex for quantum gravity, Phys. Rev. D 76 (2007), 084028, 14 pages, arXiv:0705.0674.

[70] Livine E.R., Tambornino J., Spinor representation for loop quantum gravity, J. Math. Phys. 53 (2012), 012503, 33 pages, arXiv:1105.3385.

[71] Magliaro E., Marcianó A., Perini C., Coherent states for FLRW space-times in loop quantum gravity, Phys. Rev. D 83 (2011), 044029, 9 pages, arXiv:1011.5676.

[72] Magliaro E., Perini C., Local spin foams, arXiv:1010.5227.

[73] Magliaro E., Perini C., Regge gravity from spinfoams, arXiv:1105.0216. 
[74] Morales-Técotl H.A., Rovelli C., Fermions in quantum gravity, Phys. Rev. Lett. 72 (1994), 3642-3645, gr-qc/9401011.

[75] Morales-Técotl H.A., Rovelli C., Loop space representation of quantum fermions and gravity, Nuclear Phys. B 451 (1995), 325-361.

[76] Penrose R., Applications of negative dimensional tensors, in Combinatorial Mathematics and its Applications (Oxford, 1969), Academic Press, London, 1971, 221-244.

[77] Pereira R., Lorentzian loop quantum gravity vertex amplitude, Classical Quantum Gravity 25 (2008), 085013, 8 pages, arXiv:0710.5043.

[78] Perinia C., Rovellia C., Speziale S., Self-energy and vertex radiative corrections in LQG, Phys. Lett. B 682 (2009), 78-84, arXiv:0810.1714.

[79] Regge T., General relativity without coordinates, Nuovo Cim. 19 (1961), 558-571.

[80] Röken C., Private communication, 2012.

[81] Rovelli C., A new look at loop quantum gravity, Classical Quantum Gravity 28 (2011), 114005, 24 pages, arXiv:1004.1780.

[82] Rovelli C., Discretizing parametrized systems: the magic of Ditt-invariance, arXiv:1107.2310.

[83] Rovelli C., On the structure of a background independent quantum theory: Hamilton function, transition amplitudes, classical limit and continuous limit, arXiv:1108.0832.

[84] Rovelli C., Partial observables, Phys. Rev. D 65 (2002), 124013, 8 pages, gr-qc/0110035.

[85] Rovelli C., Quantum gravity, Cambridge Monographs on Mathematical Physics, Cambridge University Press, Cambridge, 2004.

[86] Rovelli C., Quantum reference systems, Classical Quantum Gravity 8 (1991), 317-331.

[87] Rovelli C., Simple model for quantum general relativity from loop quantum gravity, J. Phys. Conf. Ser. 314 (2011), 012006, 12 pages, arXiv:1010.1939.

[88] Rovelli C., What is observable in classical and quantum gravity?, Classical Quantum Gravity 8 (1991), $297-316$.

[89] Rovelli C., Zakopane lectures on loop gravity, arXiv:1102.3660.

[90] Rovelli C., Smolin L., Knot theory and quantum gravity, Phys. Rev. Lett. 61 (1988), 1155-1158.

[91] Rovelli C., Smolin L., Loop space representation of quantum general relativity, Nuclear Phys. B 331 (1990), $80-152$.

[92] Rovelli C., Smolin L., Spin networks and quantum gravity, Phys. Rev. D 52 (1995), 5743-5759, gr-qc/9505006.

[93] Rovelli C., Smolin L., The physical Hamiltonian in nonperturbative quantum gravity, Phys. Rev. Lett. 72 (1994), 446-449, gr-qc/9308002.

[94] Rovelli C., Speziale S., Geometry of loop quantum gravity on a graph, Phys. Rev. D 82 (2010), 044018, 6 pages, arXiv:1005.2927.

[95] Rovelli C., Speziale S., Lorentz covariance of loop quantum gravity, Phys. Rev. D 83 (2011), 104029, 6 pages, arXiv:1012.1739.

[96] Rovelli C., Vidotto F., Stepping out of homogeneity in loop quantum cosmology, Classical Quantum Gravity 25 (2008), 225024, 16 pages, arXiv:0805.4585.

[97] Sahlmann H., Thiemann T., Winkler O., Coherent states for canonical quantum general relativity and the infinite tensor product extension, Nuclear Phys. B 606 (2001), 401-440, gr-qc/0102038.

[98] Thiemann T., Complexifier coherent states for quantum general relativity, Classical Quantum Gravity 23 (2006), 2063-2117, gr-qc/0206037.

[99] Thiemann T., Gauge field theory coherent states (GCS). I. General properties, Classical Quantum Gravity 18 (2001), 2025-2064, hep-th/0005233.

[100] Thiemann T., Introduction to modern canonical quantum general relativity, gr-qc/0110034.

[101] Thiemann T., Lectures on loop quantum gravity, in Quantum gravity, Lecture Notes in Phys., Vol. 631, Springer, Berlin, 2003, 41-135, gr-qc/0210094.

[102] Thiemann T., Modern canonical quantum general relativity, Cambridge Monographs on Mathematical Physics, Cambridge University Press, Cambridge, 2007. 
[103] Thiemann T., Quantum spin dynamics (QSD), Classical Quantum Gravity 15 (1998), 839-873, gr-qc/9606089.

[104] Thiemann T., Winkler O., Gauge field theory coherent states (GCS). II. Peakedness properties, Classical Quantum Gravity 18 (2001), 2561-2636, hep-th/0005237.

[105] Thiemann T., Winkler O., Gauge field theory coherent states (GCS). III. Ehrenfest theorems, Classical Quantum Gravity 18 (2001), 4629-4681, hep-th/0005234.

[106] Thiemann T., Winkler O., Gauge field theory coherent states (GCS). IV. Infinite tensor product and thermodynamical limit, Classical Quantum Gravity 18 (2001), 4997-5053, hep-th/0005235.

[107] Vidotto F., Many-node/many-link spinfoam: the homogeneous and isotropic case, Classical Quantum Gravity 28 (2011), 245005, 11 pages, arXiv:1107.2633.

[108] Vidotto F., Spinfoam cosmology, J. Phys. Conf. Ser. 314 (2011), 012049, 4 pages, arXiv:1011.4705.

[109] Wilson-Ewing E., Loop quantum cosmology of Bianchi type IX models, Phys. Rev. D 82 (2010), 043508, 13 pages, arXiv:1005.5565. 